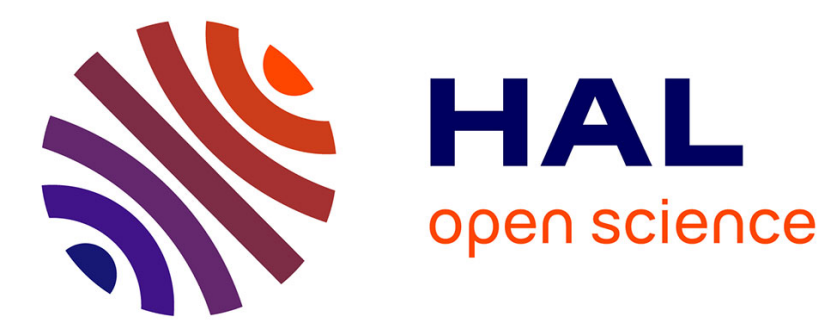

\title{
Geometric properties of a class of piecewise affine biological network models
}

\author{
Etienne Farcot
}

\section{To cite this version:}

Etienne Farcot. Geometric properties of a class of piecewise affine biological network models. Journal of Mathematical Biology, 2006, 52 (3), pp.373-418. 10.1007/s00285-005-0360-4 . hal-00830063

\section{HAL Id: hal-00830063 https://hal.inria.fr/hal-00830063}

Submitted on 4 Jun 2013

HAL is a multi-disciplinary open access archive for the deposit and dissemination of scientific research documents, whether they are published or not. The documents may come from teaching and research institutions in France or abroad, or from public or private research centers.
L'archive ouverte pluridisciplinaire HAL, est destinée au dépôt et à la diffusion de documents scientifiques de niveau recherche, publiés ou non, émanant des établissements d'enseignement et de recherche français ou étrangers, des laboratoires publics ou privés. 
Etienne Farcot

\title{
Geometric properties of a class of piecewise affine biological network models
}

\author{
Received: 13 April 2005 / Revised version: 9 September 2005/
}

Published online: 28 December 2005 - (C) Springer-Verlag 2005

\begin{abstract}
The purpose of this report is to investigate some dynamical properties common to several biological systems. A model is chosen, which consists of a system of piecewise affine differential equations. Such a model has been previously studied in the context of gene regulation and neural networks, as well as biochemical kinetics. Unlike most of these studies, nonuniform decay rates and several thresholds per variable are assumed, thus considering a more realistic model. This model is investigated with the aid of a geometric formalism. We first provide an analysis of a continuous-space, discrete-time dynamical system equivalent to the initial one, by the way of a transition map. This is similar to former studies. Especially, the analysis of periodic trajectories is carried out in the case of multiple thresholds, thus extending previous results, which all concerned the restricted case of binary systems.

The piecewise affine structure of such models is then used to provide a partition of the phase space, in terms of explicit cells. Allowed transitions between these cells define a language on a finite alphabet. Some words are proved to be forbidden in this language, thus improving the knowledge on such systems in terms of symbolic dynamics. More precisely, we show that taking these forbidden words into account leads to a dynamical system with strictly lower topological entropy. This holds for a class of systems, characterized by the presence of a splitting box, with additional conditions. We conclude after an illustrative three-dimensional example.
\end{abstract}

\section{Introduction}

Many biological systems may be described as assemblies of similar constituents evolving in parallel, and interacting in a structured way. The structure of interactions is currently modeled by an oriented graph, whose vertices represent elements in the system. Each edge represents a direct action of its initial vertex on its terminal vertex. Although very complex, and studied in its own right [1], this structure is static, and does not suffice to understand the behaviour of the whole system. Moreover, it may evolve itself as the elements in the system are changing with time. Thus, dynamical models are necessary in order to capture significant aspects of complex biological phenomena. Chosing to restrict our attention on deterministic models, two main types of formulation can be distinguished : models with

E. Farcot: COMORE INRIA, U.R. Sophia Antipolis, 2004 route des Lucioles, BP93, 06902 Sophia Antipolis, France

e-mail: etienne. farcotesophia.inria.fr

Key words or phrases: Gene and neural networks - Piecewise-affine dynamical systems Symbolic dynamics 
discrete state space $[28,40,8]$, and models with continuous state space, formulated as ordinary differential equations $[5,36,37]$. Since the latter lead to very complex nonlinear dynamics in high-dimensional spaces, and the first one only provides large scale qualitative insights about the phenomenology of the systems, intermediary formulation are often considered. Namely, systems of piecewise affine differential equations are more tractable than nonlinear smooth ones, due to their underlying discrete structure, while they yield finer information than purely discrete representations. Furthermore, they seem well suited to experimental data, which is often quantitative with non negligible uncertainty, i.e. data is partially qualitative.

The literature about the piecewise linear approach of complex nonlinear phenomena is huge, and we shall only mention typical works in the field of biology, thus ignoring many aspects such as control theoretic issues, or the many examples occuring in the context of automatics, electric and electronic circuits, or embedded software. The main reason for excluding all these very actively studied problems, is that they generally deal with complex couplings, which would not be suitably described by the class of models considered in this paper. Actually, the specificity of this class lies in the fact that the proper linear terms are uncoupled, and that interactions are only present in the piecewise constant terms of the equations. This may sound quite restrictive in regard to the much more general class of piecewise linear differential equations.

Anyway, a lot of phenomena in biology are characterized by strongly localized coupling, that is by interactions of an almost on-off nature. This includes switching networks like gene transcriptional regulation networks $[6,7,18,19,26]$, neuron networks $[17,16,30]$, as well as metabolic and chemical pathways [20], which all are currently studied examples in mathematical models of biological dynamics. Neural, metabolic and gene networks models can generally be put in the form of a system of piecewise affine differential equations, with a diagonal matrix as proper linear term. This class of model has been investigated in itself, without specially focusing on one of the different phenomena we just mentioned [10,23,38, 17]. Such a class could be called continuous-time switching networks, but this does not illustrate the piecewise linearity of the equations, and would enclose a broader range of models. The term Glass networks has been proposed in [10], which seems appropriate, since Leon Glass is the first author to have explicitly proposed this model, emphasizing its usefulness as tractable indicator of the qualitative properties of nonlinear biological systems. Although the equations studied here are more general than what is usually called a Glass system, this term will sometimes be used in the sequel. We will use the term binary systems when referring to systems with only one threshold per variable (i.e. two discrete states, whence the appellation).

The main contribution of this paper lies in the fact that working hypotheses are lightened as compared to previous studies, such as $[10,16,17,19-22,26,30$, 32]. Namely, several thresholds are allowed for each variable in the system all through this work, and degradation rates are not supposed uniform for most of our results. Thus we deal with a more realistic model than what is usually done, which leads to mathematical complications. To circumvent these difficulties, we 
adopt a geometric point of view on the dynamics. This approach proves useful with respect to the analysis of periodic orbits, as well as the symbolic dynamics approach.

On the other hand, a noticeable restriction of the present work is that it concerns networks without autoregulation. Although severe in terms of biological plausibility, this assumption is made in all studies cited above. Actually, solutions are not well defined in systems with autoregulation. Two techniques can be found in the literature to face this difficulty. The first one consists in studying a smooth dynamical system with sigmoids, which tends to the piecewise affine system in the limit of infinite steepness. The analysis can be then carried out using singular perturbation techniques $[36,37]$. The second way to handle this situation, developed in $[6,23]$, is to use the Filippov notion of solution for differential equations with discontinuous right-hand side. Such solutions are defined by the way of differential inclusions, i.e. they are set-valued. As both techniques are still a current research topic, it seems reasonable to exclude the situations where they are required.

Furthermore, even with this simplifying assumption, there is still a number of open problems concerning the phase portraits of these high-dimensional dynamical systems. In short, the difficulties concern the number and localization of attractors, as well as their formal description, especially in the case of chaotic dynamics $[2,9,11,19]$. Such problems are not only of mathematical interest, and their interpretation improves the understanding of complex biological networks. For example, it is commonly assumed, since early suggestions of Waddington and Delbrück, that the attractors of a dynamical system like those we study here are the mathematical version of modes of behaviour of living cells. In particular, cell differenciation is interpreted as the coexistence of multiple attractors with large basins of attraction (see the chapter XII of [39] for a discussion about this idea and its origins). Then, different cell types correspond to cells having reached different basins during the development of an organism. In the present paper, we mainly focus on the formal description of attractors, in a quite general and abstract setting. The high number of dimension of systems like gene regulation networks requires theoretical tools and results, and must be essentially qualitative. In this direction, the comparison of piecewise affine and discrete systems seems well adapted, and is a major motivation of the work presented in this paper.

In section 2, we present the model that motivated this study, and show how it can be reduced to a discrete-time dynamical system. The latter is rigorously defined in section 3 . Then, section 4 is mainly devoted to the study of periodic orbits, about which previously known properties are generalized to the multiple thresholds context. Finally, section 5 concerns symbolic dynamics of the studied system. The latter is defined formally, and topological entropies of different codings are compared. As a main result, it is shown that the topological entropy of purely discrete models is strictly greater than that induced by a coding of piecewise affine dynamics. This inequality holds regardless of precise parameter values, and for a large class of systems characterized by the presence of a splitting box. This result is illustrated on a three-dimensional example in section 5.3. 


\section{Model description}

\subsection{Equations}

The general model studied here is a system of differential equations of the form :

$$
\frac{d x}{d t}=\Gamma(x)-\Lambda x
$$

where $x \in \mathbb{R}^{n}, \Gamma: \mathbb{R}^{n} \rightarrow \mathbb{R}^{n}$ is piecewise constant, and $\Lambda \in \mathbb{R}_{+}^{n \times n}$ is diagonal. We note $\Gamma=\left(\gamma_{1} \ldots \gamma_{n}\right)$, where $\gamma_{i}: \mathbb{R}^{n} \rightarrow \mathbb{R}$, and $\Lambda_{i i}=\lambda_{i}$.

Each coordinate $x_{i}$ of vector $x$ represents a characteristic quantity of the $i$ th member in a finite population of $n$ interacting elements. For example, $x_{i}$ is the concentration of a protein whose production is induced from gene $i$, or $x_{i}$ is the voltage of a single neuron $i$. Each quantity in this kind of model is bounded, so that the domain in phase space where dynamics must be confined will be the cube $\mathcal{U}=[0,1]^{n}$.

Non-diagonal elements of $\Lambda$ being zero, this matrix only describes linear degradation of every component in the network. Thus, $\Lambda$ is not related to coupling or autoregulation, but rather to the fact that biological systems are usually dissipative, i.e. state space is globally contracted by the flow. Then, each diagonal element of $\Lambda$ is supposed positive.

On the other hand, $\Gamma$ describes coupling in the system. As it is piecewise constant, the domain of interest $\mathcal{U}$ has to be partitioned. This will be done with $n$ rectangles, i.e. products of $n$ bounded intervals. Such regions will be called boxes, as usually done in the literature. In each box, $\Gamma$ takes a constant value, i.e. the production rate (resp. activity) of all genes (resp. neurons) in the network is constant. Hence a box is a region in phase space where the dynamics is appropriately described by a simple linear system.

This choice of a rectangular partition may seem arbitrary, or aimed at simplifying the analysis and computations. In fact it is biologically relevant, since gene activation rates are known to evolve in a switch-like manner, that is they undergo sudden changes when some regulating protein reaches a threshold value. Neuronal response to synaptic entries are also of switching nature. In this latter case, the sudden changes usually appear when a certain linear combination of the $x_{i}$ 's reaches a threshold. However, a simple change of variable leads to formulation (1), where each threshold is related to a single variable, as explained in [29].

These thresholds yield the boundaries of boxes ; we note and number them

$$
\Theta_{i}=\left\{\theta_{i j} \mid j \in \mathbb{N}_{p_{i}}\right\}
$$

in each direction $i$, with the notation $\mathbb{N}_{p_{i}}=\left\{1 \cdots p_{i}\right\}$.

For the sake of biological consistency, when (1) models a gene network, the $\theta_{i j}$ 's are often given in an unordered way, thus focusing on the interactions between genes : $\theta_{i j}$ is the threshold at which gene $i$ activates (resp. inhibits) gene $j$ by increasing (resp. decreasing) $\gamma_{j}$ 's value when crossing this threshold from left to right [38, 40]. However, there is always a permutation $\sigma: \mathbb{N}_{p_{i}} \rightarrow \mathbb{N}_{p_{i}}$, such that the $\theta_{i \sigma(j)}$ are in increasing order. Since we care more about phase space geometric structure 
than about dependence between genes (i.e. the interaction graph), we will assume from now on, that the sets $\Theta_{i}$ are ordered : $\theta_{i 1}<\theta_{i 2}<\cdots<\theta_{i p_{i}}$.

Then, boxes are explicitly written as :

$$
B_{a}=B_{a_{1} \ldots a_{n}}=\prod_{i=1}^{n}\left[\theta_{i, a_{i}}, \theta_{i, a_{i}+1}\right],
$$

where the subscript $a$, belongs to the finite set

$$
\mathcal{A}=\prod_{i=1}^{n} \mathbb{N}_{p_{i}}
$$

Since this subscript $a$ uniquely determines a box, and since $\Gamma$ is constant in each box, it will be convenient to consider this mapping as $\Gamma: \mathcal{A} \rightarrow \mathbb{R}^{n}$.

The set $\mathcal{A}$ will sometimes be called an alphabet in the sequel. It will be convenient to note elements of $\mathcal{A}$ as strings of the form $a=a_{1} \ldots a_{n}$, instead of vectors. One advantage of using symbols in the discrete set $\mathcal{A}$ is that it underscores the qualitative nature of the model. Each symbol $a$ can be seen as a discrete state in which all characteristic quantities of the system are constant. As such, it leads to an automaton model that will be explained more deeply in section 5. Moreover, as a finite subset of the lattice $\mathbb{N}^{n}$, it inherits nice geometric features of this lattice. Especially, symbols in $\mathcal{A}$ correspond to boxes in $\mathcal{U}$, while straight lines joining these symbols correspond to facets of boxes. Hence, the geometric structure of $\mathcal{A}$ is somehow 'dual' to that of the partition of $\mathcal{U}$. This is particularly striking in $\mathbb{R}^{2}$, as illustrated on figure 1. In arbitrary dimension, this also has practical consequences that will be exploited in section 5 .

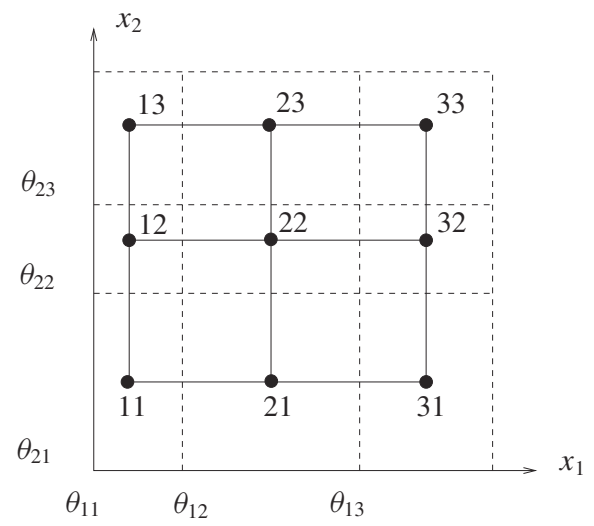

Fig. 1. An example of partition into boxes, when $\mathcal{U}=[0,1]^{2}$. There are three thresholds in both directions, hence $\mathcal{A}=\mathbb{N}_{3} \times \mathbb{N}_{3}$. After a rescaling, this set can be superposed on the box partition, so that neighbouring boxes correspond to symbols differing by \pm 1 in a single direction 


\subsection{Flow}

A system of form (1) induces a flow that can be explicitly written. In a given box $B_{a}, \Gamma$ is a constant vector, thus the flow inside this box is :

$$
\varphi^{a}(t, x)=x(t)=f+e^{-\Lambda t}(x-f) \quad \text { where } \quad f=\left(\frac{\gamma_{i}}{\lambda_{i}}\right)_{i=1 \ldots n} .
$$

Here, the vector $f$ is called a focal point, because it is obviously attracting in the above equation. Hence, depending on its position with respect to $B_{a}$, it will be an asymptotically stable steady state, or the trajectory will encounter the boundary of the box. In the latter case, $\Gamma$ is assigned a new value according to certain rules that will be made precise, and one constructs a new piece of the trajectory by continuity.

Some results about polytopes will be useful afterwards. In the rest of the paper we mainly lean on $[24,41]$ for such kind of properties. The boundary of $B_{a}$ is formed by $k$-faces, which are $k$-dimensional rectangles, for $k \in\{0 \ldots n-1\}$. When the intersection happens at a $n-1$ face, or facet (or wall), there is at most one adjacent box and the new value of $\Gamma$ is unambiguous. In the case of lower dimensional faces, there are several adjacent boxes in general. Let $k$ be the dimension of the face encountered by $\varphi^{a}(t, x)$, i.e. it is given by $n-k$ hyperplane equations of the form $x_{i}=\theta_{i j}$. There are $2^{n-k}$ adjacent boxes sharing this face, including $B_{a}$, corresponding to the above/below position with respect to each of the defining hyperplanes (on the boundary of the domain $\mathcal{U}$, there are of course less adjacent boxes). In each of these neighbouring boxes, the flow has a fixed value, for which the $k$-dimensional face can be either attracting or repelling. The resulting flow is not straightforwardly defined in such regions, as will be made precise in section 3.3. The general case requires the Filippov notion of solution of a differential equation with discontinuous right-hand side [6,23]. Until further clarifications (sec. 3.3), we can exclude all $k$-dimensional faces, for $k<n-1$, like is done for example in [17, 16].

With this procedure, one constructs continuous trajectories as far as they do not cross any face of dimension $<n-1$. There are still some degenerate cases for which this construction is not well defined. We will make two assumptions that allow us to neglect these cases. The first one is :

H1. $\forall a \in \mathcal{A}, f(a) \in \bigcup_{a \in \mathcal{A}} \operatorname{int}\left(B_{a}\right)$.

Here $f$ is considered as the map $\Lambda^{-1} \Gamma: \mathcal{A} \rightarrow \mathbb{R}^{n}$, and int denotes the interior. This assumption means that the focal points all lie inside the domain $\mathcal{U}$, and that none of them is on the boundary of a box. The first aspect implies that $\mathcal{U}$ is positively invariant, and thus can be considered as the only region where relevant dynamics take place. The second one excludes (rare) cases which would cause technical complications without improving the model.

The second hypothesis concerns the case of autoregulation. As mentioned in the introduction, this assumption is certainly too strong in regard to biological phenomena, but it may lead to the use of generalized solutions in the sense of Filippov, i.e. differential inclusions, to have a mathematically rigorous definition of the flow [6,23]. We neglect this kind of difficulty, by restricting the allowed 
dispositions of boxes and their focal points. Observe that two boxes $B_{a}$ and $B_{a^{\prime}}$ are adjacent in a single direction (i.e. through a facet) if and only if $\left\|a-a^{\prime}\right\|_{1}=1$, or equivalently if and only if there is some $i \in \mathbb{N}_{n}$ such that $a-a^{\prime}= \pm \mathbf{e}_{i}\left(\mathbf{e}_{i}\right.$ being the $i$ th vector of the canonical basis of $\mathbb{R}^{n}$ ). Using a discretizing operator $\mathbf{d}=\left(\mathbf{d}_{1} \ldots \mathbf{d}_{n}\right): \bigcup_{a} \operatorname{int}\left(B_{a}\right) \rightarrow \mathcal{A}$, which maps a point lying inside a box to the subscript of this box, we can write

H2. $\forall i \in \mathbb{N}_{n}, \forall a, a^{\prime} \in \mathcal{A}, a-a^{\prime}= \pm \mathbf{e}_{i}$,

$$
\left(\mathbf{d}_{i}(f(a))-a_{i}\right)\left(\mathbf{d}_{i}\left(f\left(a^{\prime}\right)\right)-a_{i}^{\prime}\right)>0,
$$

or

$$
\left(\mathbf{d}_{i}(f(a))-a_{i}\right)=0 \text { and }\left(\mathbf{d}_{i}\left(f\left(a^{\prime}\right)\right)-a_{i}^{\prime}\right)\left(a_{i}-a_{i}^{\prime}\right)>0,
$$

or the same with a and $a^{\prime}$ exchanged.

In other words, $\mathbf{H} 2$ means that the $i$ th component of the vector field does not change in sign when crossing a wall in direction $i$. Autoregulation (i.e. $\dot{x}_{i}$ depends on $x_{i}$ ) is a necessary, but not a sufficient condition for this configuration to happen. We thus do not reject all forms of autoregulation here. The situations avoided are schematically represented in figure 2 .

\subsection{Transition map}

Once the flow (4) is given in a box $B_{a}$, it is easy to compute the time and position at which it intersects the boundary of $B_{a}$, if ever. The possibility for each facet to be encountered by the flow depends uniquely on the position of the focal point : $\left\{x_{i}=\theta_{i, a_{i}}\right\}$ (resp. $\left\{x_{i}=\theta_{i, a_{i}+1}\right\}$ ) can be crossed if and only if $f_{i}<\theta_{i, a_{i}}$ (resp. $\left.f_{i}>\theta_{i, a_{i}+1}\right)$. According to this observation, we note

$$
I_{\text {out }}^{+}(a)=\left\{i \in \mathbb{N}_{n} \mid f_{i}>\theta_{i, a_{i}+1}\right\}, \quad \text { and } \quad I_{\text {out }}^{-}(a)=\left\{i \in \mathbb{N}_{n} \mid f_{i}<\theta_{i, a_{i}}\right\},
$$

and $I_{\text {out }}(a)=I_{\text {out }}^{+}(a) \cup I_{\text {out }}^{-}(a)$.

When it is unambiguous, we will omit the dependence on $a$, as we have already done implicitly with the focal point.
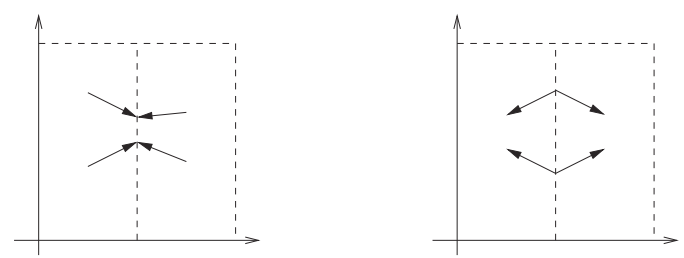

Fig. 2. The two possible ambiguities due to autoregulation, often called black wall (on the left) and white wall (on the right) 
Since these functions will be useful in the following, we note :

$$
\alpha_{i}^{-}(x)=\frac{f_{i}-\theta_{i, a_{i}}}{f_{i}-x_{i}}, \quad \text { and } \quad \alpha_{i}^{+}(x)=\frac{f_{i}-\theta_{i, a_{i}+1}}{f_{i}-x_{i}} .
$$

Now, in each direction $i \in I_{\text {out }}$ the time at which $\varphi^{a}(t, x)$ encounters the corresponding hyperplane, for $x \in B_{a}$, is given by :

$\tau_{i}(x)=\frac{-1}{\lambda_{i}} \ln \left(\alpha_{i}^{-}(x)\right)$ if $i \in I_{\text {out }}^{-}, \quad$ and $\quad \tau_{i}(x)=\frac{-1}{\lambda_{i}} \ln \left(\alpha_{i}^{+}(x)\right)$ if $i \in I_{\text {out }}^{+}$.

This distinction between directions in $I_{\text {out }}^{+}$and $I_{\text {out }}^{-}$shall occur quite often, leading to unnecessarily cumbersome discussions. Here this distinction may be avoided by observing that whenever $i \in I_{\text {out }}$,

$$
\tau_{i}(x)=\frac{-1}{\lambda_{i}} \ln \left(\alpha_{i}(x)\right) \quad \text { where } \quad \alpha_{i}(x)=\min \left(\alpha_{i}^{-}(x), \alpha_{i}^{+}(x)\right) .
$$

This can be checked by inspecting the range of $\alpha_{i}^{ \pm}$for all possible $i$ (see table (6)), and using the fact that $\tau(x)$ must be nonnegative.

\begin{tabular}{|c|c|c|}
\hline$i$ & $\alpha_{i}^{-}(x)$ & $\alpha_{i}^{+}(x)$ \\
\hline$\in I_{\text {out }}^{-}$ & $\left.\left.\left[\alpha_{i}^{-}\left(\theta_{i, a_{i}+1}\right), 1\right] \varsubsetneqq\right] 0,1\right]$ & {$\left[1, \alpha_{i}^{+}\left(\theta_{i, a_{i}}\right)\right]$} \\
$\in I_{\text {out }}^{+}$ & {$\left[1, \alpha_{i}^{-}\left(\theta_{i, a_{i}+1}\right)\right]$} & $\left.\left.\left[\alpha_{i}^{+}\left(\theta_{i, a_{i}}\right), 1\right] \varsubsetneqq\right] 0,1\right]$ \\
$\notin I_{\text {out }}$ and $x_{i}<f_{i}$ & {$[1,+\infty[$} & ]$\left.-\infty, \alpha_{i}^{+}\left(\theta_{i, a_{i}}\right)\right] \varsubsetneqq \mathbb{R}_{-}^{*}$ \\
$\notin I_{\text {out }}$ and $x_{i}>f_{i}$ & ]$\left.-\infty, \alpha_{i}^{-}\left(\theta_{i, a_{i}+1}\right)\right] \varsubsetneqq \mathbb{R}_{-}^{*}$ & {$[1,+\infty[$} \\
\hline
\end{tabular}

The directions that are not in $I_{\text {out }}$ are of no use here, but will be considered in section 3.1. Now notice that $\alpha_{i}$ is not defined if $x_{i}=f_{i}$, which may only happen for $i \notin I_{\text {out }}$.

Taking the minimum

$$
\tau(x)=\min _{i \in I_{\text {out }}} \tau_{i}(x) .
$$

and reinjecting it in equation (4), we get the exiting point of $B_{a}$ for the initial condition $x$. Since this process is intended to be repeated along trajectories, $x$ will generally lie on the boundary of the current box, except for the initial condition, which may however be chosen without loss of generality on a wall. Then we get a transition map $\mathcal{M}^{a}: \partial B_{a} \rightarrow \partial B_{a}$, which can be made explicit, omitting $a$ :

$$
\begin{aligned}
\mathcal{M} x & =\varphi(\tau(x), x) \\
& =f+A(x)(x-f) .
\end{aligned}
$$

Where $A(x)$ is the diagonal matrix whose entries are $e^{-\lambda_{i} \tau(x)}$. If $q$ is an escaping direction, i.e. $\tau=\tau_{q}$, we can also write the entries of $A(x)$ as $\left(\alpha_{q}(x)\right)^{\frac{\lambda_{i}}{\lambda q}}$, for $i=1 \ldots n$. 
We see here that $\mathcal{M}$ is nonlinear, but in the special case where

H3. $\forall i, j \in \mathbb{N}_{n}, \lambda_{i}=\lambda_{j}$, i.e. $\exists \lambda \in \mathbb{R}_{+}^{*}, \Lambda=\lambda I d$.

is fulfilled, $A(x)$ is proportional to the identity matrix as well, and $\mathcal{M}$ can be understood as a projective transformation (see e.g. [41] p.67). Actually, H3 implies that the flow in each box consists of straight lines directed towards $f$, and thus $\mathcal{M} x$ is one of the intersections of the affine line $f+\mathbb{R}(x-f)$ with $\partial B_{a}$. The set of all trajectories in $B_{a}$ is then the intersection of this box with the straight lines composing the polyhedral cone $f+\left\{t(x-f) \mid t \geqslant 0, x \in B_{a}\right\}$. These nice aspects explain why this last assumption is currently done. Formally, (8) may be rewritten quite simply under the latter assumption :

$$
\mathcal{M} x=f+\alpha(x)(x-f),
$$

where

$$
\alpha(x)=\max _{i \in I_{\text {out }}}\left\{\alpha_{i}(x)\right\} .
$$

As $\alpha_{i}(x)$ only depends on coordinate $x_{i}$, we sometimes abusively note $\alpha_{i}\left(x_{i}\right)$.

Unless indicated, we suppose in the following that assumptions $\mathbf{H 1}$ and $\mathbf{H 2}$ are satisfied. The uniform decay rates assumption $\mathbf{H 3}$ will not be systematically required, unlike most of the previous works concerning equations of the form (1). In particular, the term Glass networks usually refers to a system of the form (1), with only one threshold $\theta_{i}$ distinct from the domain's boundaries, in each direction $i$, and the two hypotheses H1, H3 (see e.g. [10,27]). More or less explicit versions of $\mathbf{H} \mathbf{2}$ are also very largely assumed in papers dealing with such models.

\section{Properties of the transition map}

\subsection{Local properties}

In each box, $I_{\text {out }}$ determines all reachable boxes. Those are adjacent to the current box through walls supported by hyperplanes of the form $\left\{x_{i}=\theta_{i j}\right\}$, for $i \in I_{\text {out }}$, and $j \in\left\{a_{i}, a_{i}+1\right\}$ depending on $i$ 's belonging to $I_{\text {out }}^{-}$or $I_{\text {out }}^{+}$. We introduce the following notation for such walls :

$$
W_{i}^{+}(a)=\left\{x \mid x_{i}=\theta_{i, a_{i}+1}\right\} \cap B_{a} \quad \text { and } \quad W_{i}^{-}(a)=\left\{x \mid x_{i}=\theta_{i, a_{i}}\right\} \cap B_{a} .
$$

Then, each box can be partitioned in $\# I_{\text {out }}$ regions from which a single adjacent box is reachable. \# denotes the cardinal. See figures 3 and 4 for an illustration.

The subscripts $a$ will be omitted in this section, where a single box $B$ is considered. Due to previous discussions, the only walls through which trajectories may escape $B$ may be put in the form $W_{i}^{+}$(resp. $W_{i}^{-}$), for $i \in I_{\text {out }}^{+}\left(\right.$resp. $i \in I_{\text {out }}^{-}$).

Conversely, any point on a wall of the form $W_{i}^{ \pm}, i \in I_{\text {out }}^{ \pm}$, escapes immediately, as can be seen from equation (7), where escaping time is clearly zero on the corresponding escaping wall.

More precisely, at any initial condition $x$, from equations (4), (7), the directions $i$ such that $\tau(x)=\tau_{i}(x)$ are exactly those for which $\mathcal{M} x \in W_{i}^{ \pm}$.

According to this, we can coarsely partition $\partial B$ into two regions : 


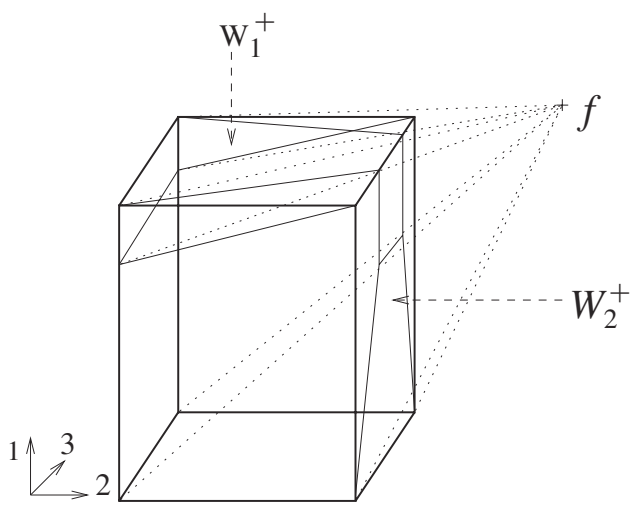

Fig. 3. A box in $\mathbb{R}^{3}$, with two escaping walls : $W_{1}^{+}$and $W_{2}^{+}$. Thus, $I_{\text {out }}=\{1,2\}$, and $\pm_{1}= \pm_{2}=+$
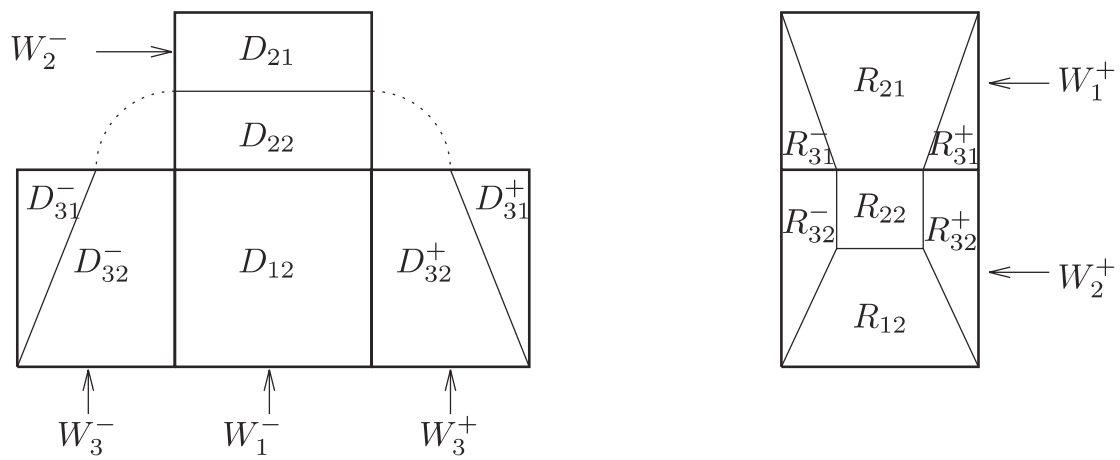

Fig. 4. Partition of $\partial B^{\text {in }}$ (on the left, seen from inside $B$ ) and $\partial B^{\text {out }}$ (on the right, seen from outside $B$ ). Dotted lines on the unfolded representation of $\partial B^{i n}$ relate points that are identical in $\mathbb{R}^{3}$. The scale is arbitrary

- the outgoing region $\partial B^{\text {out }}=\bigcup_{i \in I_{\text {out }}^{ \pm}} W_{i}^{ \pm}=\{x \in B \mid \tau(x)=0\}$

- the incoming region $\partial B^{i n}=\overline{\partial B \backslash \partial B^{\text {out }}}=\bigcup_{i \in I_{\text {out }}^{ \pm}} W_{i}^{\mp} \cup \bigcup_{i \notin I_{\text {out }}}\left(W_{i}^{-} \cup W_{i}^{+}\right)$.

Where, for any set $S, \bar{S}$ denotes its closure, and for $\pm \in\{-,+\}$ the symbol $\mp$ denotes the opposite sign. Thus, the incoming and outgoing regions are unions of walls, which are closed and cover the boundary $\partial B$. We can observe that $\partial B^{\text {out }} \cap \partial B^{\text {in }} \neq \varnothing$ whenever $\partial B^{\text {out }} \neq \varnothing$, and is the union of some $n-2$-facets of $B$. Actually, $\partial B^{i n} \neq \varnothing$ always holds, due to $i \in I_{\text {out }}^{ \pm} \Longrightarrow W_{i}^{\mp} \subset \partial B^{\text {in }}$, and $i \notin I_{\text {out }} \Longrightarrow$ $W_{i}^{-} \cup W_{i}^{+} \subset \partial B^{i n}$, as follows from $\mathbf{H 1}$.

This first partition only allows a distinction between escaping directions and the others, and one gets :

$$
\partial B^{\text {out }}=\varnothing \quad \Longleftrightarrow f \in \operatorname{int}(B),
$$


which we recall corresponds to $f$ being an asymptotically stable equilibrium point with $B$ contained in its attracting basin.

Moreover, this partition of $\partial B$ can lead to the transition map being bijective.

Proposition 1. Assume $\mathbf{H 1}$ is satisfied for a system of form (1).

Let $\mathcal{M}$ be the transition map in a box $B$, as defined in the previous section.

Restricting the domain and range of $\mathcal{M}$ to $\partial B^{\text {in }}$ and $\partial B^{\text {out }}$ respectively, and abusively keeping its name unchanged, the following is a homeomorphism, provided $\partial B^{\text {out }} \neq \varnothing$ :

$$
\mathcal{M}: \partial B^{\text {in }} \rightarrow \partial B^{\text {out }}
$$

Proof. From its definition, $\mathcal{M}: x \mapsto \varphi(\tau(x), x)$, where $\varphi$ is the flow associated to an affine dynamical system, and is continuous if and only if $\tau$ is. This function is defined as $\tau(x)=\min _{i \in I_{\text {out }}} \tau_{i}(x)$. From equation (5) each $\tau_{i}$ might only be discontinuous if $\alpha_{i}^{+}(x)$ or $\alpha_{i}^{-}(x)$ is. From table (6), both are defined and continuous on $\left[\theta_{i, a_{i}}, \theta_{i, a_{i}+1}\right]$, for $i \in I_{\text {out }}$ (furthermore, which among $\alpha_{i}^{-}(x)$ and $\alpha_{i}^{+}(x)$ is the lowest does not depend on $x$ for such $i$ ). Then, $\tau$ is continuous as the minimum of a finite set of continuous functions.

Injectivity comes from the fact that $x$ and $\mathcal{M} x$ are always on the same orbit of $\varphi$, and from monotonicity (w.r.t. time) of all coordinates $\varphi_{i}$ of this flow, along any orbit. Surjectivity will come from the construction of the inverse mapping, which is defined on $\partial B^{\text {out }}$.

Note $\tilde{\varphi}(t, x)=\varphi(-t, x)=f+e^{\Lambda t}(x-f)$ the flow in reverse time. From positivity of $\Lambda$ 's entries, we get that all trajectories given by $\widetilde{\varphi}$ diverge to $+\infty$ in each direction, and hence leave $B$ in finite time. For $x_{i}<f_{i}\left(\operatorname{resp} . x_{i}>f_{i}\right)$, $\tilde{\varphi}(t, x)$ may only encounter $W_{i}^{-}$(resp. $\left.W_{i}^{+}\right)$in direction $i$. This can happen at time $\tilde{\tau}_{i}(x)=\frac{1}{\lambda_{i}} \ln \left(\alpha_{i}^{-}(x)\right)$ (resp. $\alpha_{i}^{+}(x)$ ). Inspecting table (6) (using the fact that $\left.i \in I_{\text {out }}^{+} \Rightarrow x_{i}<f_{i}\right)$, we get a general expression :

$$
\tilde{\tau}_{i}(x)=\frac{1}{\lambda_{i}} \ln \left(\max \left(\alpha_{i}^{-}(x), \alpha_{i}^{+}(x)\right)\right) \quad \text { and } \quad \tilde{\tau}(x)=\min _{i \in \mathbb{N}_{n}}\left(\tilde{\tau}_{i}(x)\right)
$$

for the exiting time from $x$, associated with $\tilde{\varphi}$.

There is a problem when any $x_{i}=f_{i}$, where none of $\alpha_{i}^{ \pm}(x)$ is defined. Actually, for $x_{i} \rightarrow f_{i}$ (from left or right), the quantity $\max \left(\alpha_{i}^{-}(x), \alpha_{i}^{+}(x)\right)$ always tends toward $+\infty$. But for all $j \in I_{\text {out }}$ the corresponding $\tilde{\tau}_{j}$ are always bounded (see table (6)), and it is assumed here that $I_{\text {out }} \neq \varnothing$. Thus, when $x_{i} \rightarrow f_{i}$ the minimum $\tilde{\tau}$ cannot be given by $\tilde{\tau}_{i}$.

Hence, $\mathcal{M}^{-1} x=\tilde{\varphi}(\tilde{\tau}(x), x)=\varphi(-\tilde{\tau}(x), x)$ is a continuous function, which is obviously defined on $\partial B^{\text {out }}$.

Observe that the incoming region can now be defined as $\partial B^{i n}=\{x \in B \mid \widetilde{\tau}(x)=$ $0\}$.

In the special case where $\mathbf{H 3}$ is also fulfilled, the inverse mapping can be expressed as :

$$
\mathcal{M}^{-1} y=f+\beta(y)(y-f) \text {. }
$$


where

$$
\forall i \in \mathbb{N}_{n}, \beta_{i}(y)=\max \left(\alpha_{i}^{-}(y), \alpha_{i}^{+}(y)\right), \quad \text { and } \quad \beta(y)=\min _{i \in \mathbb{N}_{n}}\left\{\beta_{i}(y)\right\} .
$$

This results from the definition, $\mathcal{M}^{-1} x=\varphi(-\tilde{\tau}(x), x)$, with $\Lambda$ proportional to the identity.

\subsection{Partition of boxes}

In this section, we shall analyze in some detail the possible configurations that may happen at a single box, in terms of incoming facets, outgoing facets, and pieces of trajectories between them. Thus, we consider a single box $B$, noted without subscript for sake of readability. Moreover, we assume that this box $B$ has at least one escaping direction, i.e. $I_{\text {out }} \neq \varnothing$, since otherwise the analysis is of little interest.

Considering a single box could seem much too local, as compared with full orbits, crossing a possibly high number of successive boxes. But at this local scale, we provide a geometric description of all possible situations, showing by the way that they form a combinatorially non trivial set. This description relies on a finer partition of $B$ than that of the previous section.

The proposed partition arises by distinguishing not only which points escape in each direction of $I_{\text {out }}$ but, on any incoming wall, which points are mapped onto a given escaping wall. Conversely, those point on an escaping wall that are mapped backwards on a given incoming wall will be distinguished.

Escaping walls are of the form $W_{j}^{ \pm}$, for $j \in I_{\text {out }}$, where \pm has a fixed value for each $j$. Thus, we note $\pm_{j}$ the unique sign such that $j \in I_{\text {out }}^{ \pm_{j}}$. The somehow unusual symbol $\mp j$ is then defined as the opposite of $\pm_{j}$, and will be useful on more than one occasion. One may observe that $\pm_{j}=\operatorname{sign}\left(\mathbf{d}_{j}(f(a))-a_{j}\right)$.

Since we deal with a single box, a non ambiguous and convenient notation for thresholds in this section will be : $\theta_{i}^{-} \doteq \theta_{i, a_{i}}$ and $\theta_{i}^{+} \doteq \theta_{i, a_{i}+1}$. It follows that, for $i \in I_{\text {out }}, \theta_{i}^{ \pm_{i}}$ is the single threshold that may be reached in direction $i$.

The sets we have described in words can be formalized :

$$
\begin{aligned}
& D_{i j}^{ \pm}=W_{i}^{ \pm} \bigcap \mathcal{M}^{-1}\left(W_{j}^{ \pm j}\right) \text { for } i \in \mathbb{N}_{n} \backslash I_{\text {out }}, j \in I_{\text {out }} . \\
& D_{i j}=W_{i}^{\mp_{i}} \bigcap \mathcal{M}^{-1}\left(W_{j}^{ \pm j}\right) \text { for } i, j \in I_{\text {out }} .
\end{aligned}
$$

and

$$
\begin{aligned}
& R_{i j}^{ \pm}=\mathcal{M}\left(W_{i}^{ \pm}\right) \bigcap W_{j}^{ \pm_{j}} \text { for } i \in \mathbb{N}_{n} \backslash I_{\text {out }}, j \in I_{\text {out }} . \\
& R_{i j}=\mathcal{M}\left(W_{i}^{\mp_{i}}\right) \bigcap W_{j}^{ \pm_{j}} \text { for } i, j \in I_{\text {out }} .
\end{aligned}
$$

To help memory, note that the letter $D$ stands for domain, while $R$ stands for range (of $\mathcal{M}$ ).

When $i \notin I_{\text {out }}$, both walls $W_{i}^{+}$and $W_{i}^{-}$are subsets of $\partial B^{i n}$. Accordingly, the superscript \pm above means that two sets are defined. For $i \in I_{\text {out }}$, there is no possible ambiguity in the superscripts of walls : $W_{i}^{ \pm_{i}} \subset \partial B^{\text {out }}$ and $W_{i}^{\mp i} \subset \partial B^{i n}$. 


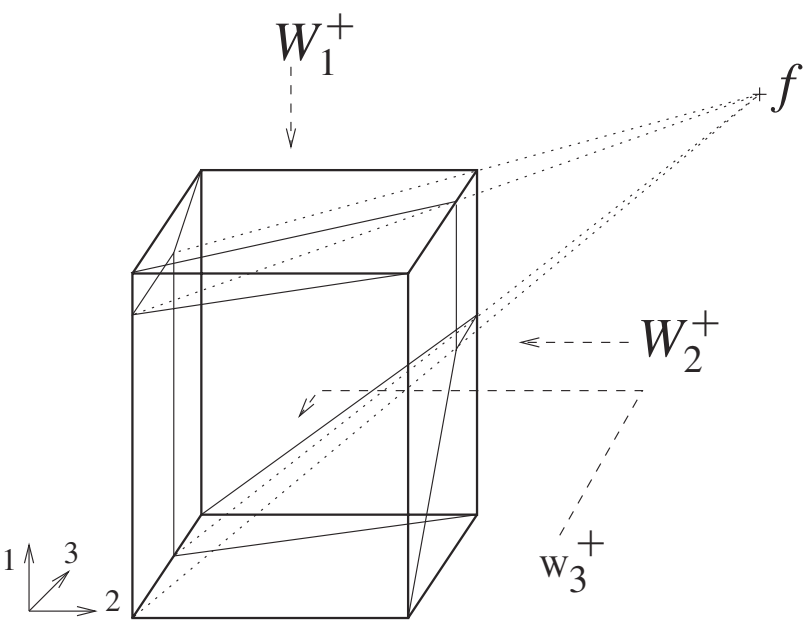

Fig. 5. A box in $\mathbb{R}^{3}$, with three escaping walls : $W_{1}^{+}, W_{2}^{+}$and $W_{3}^{+}$. Dotted lines relate the vertices of $D_{22}$ and their images, i.e. the vertices of $R_{22}$
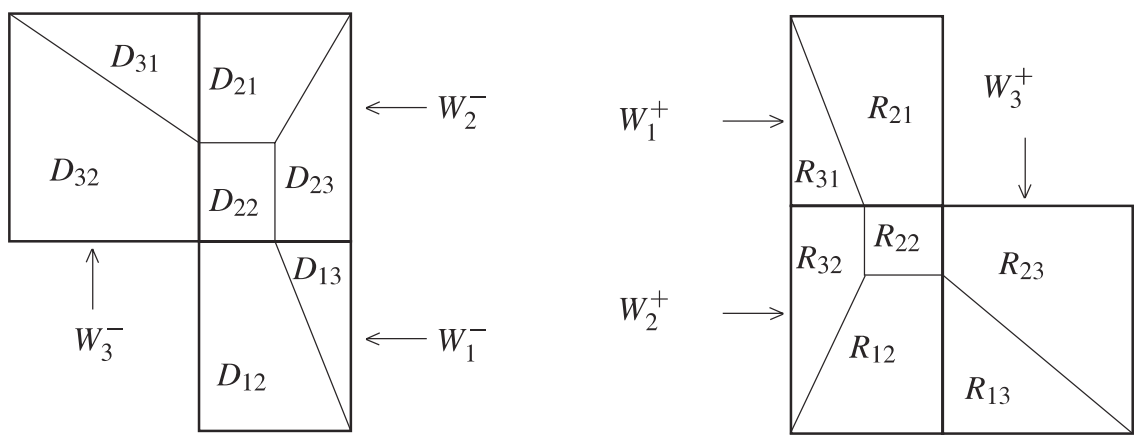

Fig. 6. Partition of $\partial B^{\text {in }}$ (on the left, seen from inside the box) and $\partial B^{\text {out }}$ (on the right, seen from outside the box). The scale is arbitrary, but the shape and position of each set is well represented

Examples of such sets are depicted in figures 3 and 4, as well as figures 5 and 6, on 3-dimensional examples, with the uniform decay rate assumption $\mathbf{H 3}$ (so that these regions are polytopes).

The fact that these sets form a partition of $\partial B$ is due to $\mathcal{M}$ being a bijection. Actually, from this we get that each point on a wall $W_{i}^{ \pm} \subset \partial B^{i n}$ must be mapped on a wall in $\partial B^{\text {out }}$, of the form $W_{j}^{ \pm_{j}}$, hence the sets $D_{i j}^{\varepsilon}$, with $\varepsilon \in\{,+,-\}$ partition $\partial B^{i n}$. Conversely each point on $\partial B^{\text {out }}$ has an antecedent on a wall of the form $W_{i}^{ \pm} \subset \partial B^{i n}$, so that $\partial B^{\text {out }}$ is partitioned by the sets $R_{i j}^{\varepsilon}$.

We now consider some properties of these sets. First, it appears that there is a simple relation between sets in (14) and in (15). 


\section{Proposition 2.}

$$
\begin{aligned}
& R_{i j}^{ \pm}=\mathcal{M}\left(D_{i j}^{ \pm}\right) \text {for } i \in \mathbb{N}_{n} \backslash I_{\text {out }}, j \in I_{\text {out }} . \\
& R_{i j}=\mathcal{M}\left(D_{i j}\right) \text { for } i, j \in I_{\text {out }} .
\end{aligned}
$$

Moreover, when $\mathbf{H 3}$ is true, the polytopal complexes formed respectively with all $D_{i j}^{\varepsilon}$ and all $R_{i j}^{\varepsilon}$ are combinatorially isomorphic. In particular, each pair of $D_{i j}^{\varepsilon}$ and $R_{i j}^{\varepsilon}$ are combinatorially isomorphic polytopes.

Proof. Both equalities follow from the fact that $\mathcal{M}: \partial B^{\text {in }} \rightarrow \partial B^{\text {out }}$ is a bijection. Actually, the injectivity of $\mathcal{M}$ implies that $\mathcal{M}(A \cap B)=\mathcal{M}(A) \cap \mathcal{M}(B)$, for all subsets $A$ and $B$ in the domain of $\mathcal{M}$. Surjectivity, on the other hand, implies $\mathcal{M}\left(\mathcal{M}^{-1}(A)\right)=A$ for any subset $A$ in the range of $\mathcal{M}$. The conclusion is a direct consequence of definitions (14) and (15).

Under H3, we have already seen that all trajectories in a box are straight lines, and that $\mathcal{M}$ is a projective transformation. Since walls are polytopes $((n-1)$-rectangles), and projective transformations transform polytopes into polytopes [24,41], the sets defined in (14) and (15) are polytopes.

The combinatorial isomorphism means that the collection of $D_{i j}^{\varepsilon}$ and that of $R_{i j}^{\varepsilon}$ have the same face structure, in terms of incidence between faces of all dimensions. This can be seen by observing that $\mathcal{M}$ is the projective mapping used in the construction of the so called Schlegel diagram, which preserves combinatorial structure (see [41], p.132-137). More precisely, for a fixed $j$, the complex formed of the $R_{i j}^{\varepsilon}$ and their subfaces is precisely the Schlegel diagram of the polytope $B \cap \mathcal{M}^{-1}\left(W_{j}^{ \pm_{j}}\right)$ based on the face $W_{j}^{ \pm_{j}}$. The polytopes $B \cap \mathcal{M}^{-1}\left(W_{j}^{ \pm_{j}}\right), j \in I_{\text {out }}$, partition $B$, and their facets are exactly $W_{j}^{ \pm_{j}}$, and all $D_{i j}^{\varepsilon}$, from which the isomorphism's construction follows.

A more thorough discussion of these constructions can be found in [14].

The sets that need a superscript + or - are also simply related :

Proposition 3. $D_{i j}^{+}=D_{i j}^{-}+\left(\theta_{i}^{+}-\theta_{i}^{-}\right) \mathbf{e}_{i}$, where $\mathbf{e}_{i}$ is the ith vector of the canonical basis.

Proof. Let $x \in W_{i}^{-}$, and define $x^{\prime} \doteq x+\left(\theta_{i}^{+}-\theta_{i}^{-}\right)$.

We show now that $x$ belongs to $D_{i j}^{-}$if and only if $x^{\prime}$ belongs to $D_{i j}^{+}$.

$x \in D_{i j}^{-}$if and only if the orbit starting at $x$ escapes in direction $j$, i.e. $\tau(x)=$ $\tau_{j}(x)$. Since $x$ and $x^{\prime}$ have identical coordinates, except $x_{i}=\theta_{i}^{-}$and $x_{i}^{\prime}=\theta_{i}^{+}$, with $i \notin I_{\text {out }}$ (see (14)), the exit time $\tau(x)$ is independent of $x_{i}$. Hence $\tau(x)=\tau\left(x^{\prime}\right)=$ $\tau_{j}\left(x^{\prime}\right)$, or equivalently $\mathcal{M} x^{\prime} \in W_{j}^{ \pm_{j}}$, i.e. $x^{\prime} \in D_{i j}^{+}$.

Now a result restricts the possible transitions between incoming and outgoing walls. The term relint denotes the relative interior : for any set $S$ relint $(S)$ is the interior taken in the smallest affine subspace containing $S$. In the rest of the text, interior will mean relative interior.

Lemma 1. There is at most one $i \in I_{\text {out }}$, such that $\operatorname{relint}\left(D_{i i}\right)$ (and thus $\operatorname{relint}\left(R_{i i}\right)$ ) is nonempty. 
Proof. Let $i \in \mathbb{N}_{n}$ such that $\operatorname{relint}\left(D_{i i}\right) \neq \varnothing$. Equivalently, there exists an $x^{*}$ in $W_{i}^{\mp i}$, such that $\mathcal{M} x^{*} \in W_{i}^{ \pm_{i}}$. The first condition implies $x_{i}^{*}=\theta_{i}^{\mp i}$, while the second implies that $\tau\left(x^{*}\right)=\tau_{i}\left(x_{i}^{*}\right)=\tau_{i}\left(\theta_{i}^{\mp i}\right)$ is a minimum. $x^{*}$ being in the relative interior of $D_{i i}$, this minimum is strict :

$$
\forall k \in I_{\text {out }}, k \neq i, \quad \tau_{i}\left(x_{i}^{*}\right)<\tau_{k}\left(x_{k}^{*}\right) .
$$

Now, from table (6) and monotonicity of $\alpha_{i}^{ \pm}$functions, it follows that $\tau_{i}\left(\theta_{i}^{\mp i}\right)$ is the maximum value of all $\tau_{i}\left(x_{i}\right)$, for $x_{i} \in\left[\theta_{i}^{-}, \theta_{i}^{+}\right]$. Actually, $\alpha_{i}\left(x_{i}\right)$ has a minimum at $x_{i}=\theta_{i}^{\mp i}$, and $\tau_{i}$ decreases with respect to $\alpha_{i}$. Thus one gets :

$$
\forall i \in I_{\text {out }}, \quad \tau_{i}\left(x_{i}^{*}\right)=\tau_{i}\left(\theta_{i}^{\mp i}\right)=\max _{x_{i} \in\left[\theta_{i}^{-}, \theta_{i}^{+}\right]} \tau_{i}\left(x_{i}\right)
$$

From the latter and (16) we derive a necessary condition for relint $\left(D_{i i}\right) \neq \varnothing$ :

$$
\forall k \in I_{\text {out }}, k \neq i, \quad \max _{x_{i} \in\left[\theta_{i}^{-}, \theta_{i}^{+}\right]} \tau_{i}\left(x_{i}\right)<\max _{x_{k} \in\left[\theta_{k}^{-}, \theta_{k}^{+}\right]} \tau_{k}\left(x_{k}\right),
$$

which obviously cannot be satisfied by more than one $i \in I_{\text {out }}$.

Remark 1. From lemma 1, the number of directions such that $D_{i i}$ has nonempty interior is 0 or 1 . The case where it is 0 is rare, in the sense that there is a measure zero set of parameters leading to it. This set is given by the equality of all maximal values of the $\tau_{i}$ functions (which occur at threshold values of $x_{i}$ ), for $i \in I_{\text {out }}$ and $\# I_{\text {out }} \geqslant 2$.

When $\# I_{\text {out }} \in\{0,1\}$, this number is equal to the number of directions $i$ with nonempty $\operatorname{relint}\left(D_{i i}\right)$.

The previous lemma indicates that not all transitions are admissible for a fixed set of parameters. The next result shows that there are no other restrictions of this kind.

Lemma 2. For $i \in \mathbb{N}_{n}, j \in I_{\text {out }}, i \neq j$, and $\varepsilon \in\{,+,-\}$, all $D_{i j}^{\varepsilon}$, (resp. $\left.R_{i j}^{\varepsilon}\right)$, have nonempty relative interior.

Proof. For $i \notin I_{\text {out }}$, from proposition $3, D_{i j}^{-}$and $D_{i j}^{+}$are obtained from each other by a translation, so that it is sufficient to consider only one of them. Recall that $\mp i$ is only defined for $i \in I_{\text {out }}$. A practical convention, for $i \notin I_{\text {out }}$, will be to define $\mp_{i}$ as being any of the two signs,-+ . Then $\mp_{i}$ being well-defined for $i \in \mathbb{N}_{n}$, the result will follow if we show that

$$
W_{i}^{\mp i} \cap \mathcal{M}^{-1}\left(W_{j}^{ \pm_{j}}\right)
$$

is of nonempty interior, for $i \in \mathbb{N}_{n}$ and $j \in I_{\text {out }}$.

Again, and whatever $i, \theta_{i}^{\mp i}$ is a generic notation that will be useful to avoid enumerating several analogous cases.

The interior of a set like (17) is defined by the equality $x_{i}=\theta_{i}^{\mp i}$, and the inequalities $\tau_{j}\left(x_{j}\right)<\tau_{k}\left(x_{k}\right)$ for $k \in I_{\text {out }} \backslash\{j\}$.

From table (6) it appears that the range of the $\alpha_{k}$ functions always takes the form $\left[\alpha_{k}^{ \pm_{k}}\left(\theta_{k}^{\mp k}\right), 1\right]$, when $k \in I_{\text {out }}^{ \pm_{k}}$. The upper value 1 is given by $\alpha_{k}^{ \pm_{k}}\left(\theta_{k}^{ \pm_{k}}\right)=1$. 
Thresholds being distinct in each direction, the range of any $\alpha_{k}$ is of nonempty interior. Then, each $\tau_{k}$ has also a range of nonempty interior, given by $\left[0, \frac{-1}{\lambda_{k}} \ln \left(\alpha_{k}^{ \pm_{k}}\left(\theta_{k}^{\mp k}\right)\right)\right]$. If

$$
\tau^{*}=\min _{k \in I_{\text {out }}} \frac{-1}{\lambda_{k}} \ln \left(\alpha_{k}^{ \pm_{k}}\left(\theta_{k}^{\mp k}\right)\right),
$$

one gets a positive length interval $\left[0, \tau^{*}\right]$, which is contained in the range of all $\tau_{k}$, for $k \in I_{\text {out }}$. Since $\# I_{\text {out }}$ is finite, we can choose this number of points in the latter interval $: \vartheta_{1}>\cdots>\vartheta_{\# I_{\text {out }}}$. Then, we construct a point $x$ as follows:

- $x_{i}=\theta_{i}^{\mp i}$

- $x_{j}=\tau_{j}^{-1}\left(\vartheta_{\# I_{\text {out }}}\right)$, which is possible by definition of the $\vartheta_{k}$ 's,

- $x_{k}=\tau_{k}^{-1}\left(\vartheta_{p}\right)$ for $k \in I_{\text {out }} \backslash\{j\}$, where $p$ is arbitrary in $\left\{1 \ldots \# I_{\text {out }}-1\right\}$,

- $\left.x_{k} \in\right] \theta_{k}^{-}, \theta_{k}^{+}\left[\right.$for $k \in \mathbb{N}_{n} \backslash I_{\text {out }}$.

It is clear then, that this point belongs to the relative interior of the set (17).

Remark 2. The fact that $i \neq j$, which makes the difference with lemma 1 , is important here. It is implicitly useful for it allows one to choose $x_{j}$ in the whole domain $\left[\theta_{j}^{-}, \theta_{j}^{+}\right]$, while $x_{i}$ is of fixed value $\theta_{i}^{\mp i}$.

To conclude this section, we shall in fact provide an explicit description of the nonempty regions $D_{i j}^{\varepsilon}$ and $R_{i j}^{\varepsilon}$. This description can be useful from an algorithmic point of view, since it is a minimal set of inequalities, hence optimal in terms of memory resources.

Proposition 4. For all but a zero-measure set of parameters (i.e. thresholds and focal point coordinates) the sets $D_{i j}^{\varepsilon}$ defined in eq. (14), are bounded cells with piecewise smooth boundary, whose (relative) interior is irredundantly defined by the following inequalities:

$$
\begin{cases}x_{i}=\theta_{i}^{\varepsilon}, & \\ \theta_{k}^{-}<x_{k}<\theta_{k}^{+}, & k \in\left(\mathbb{N}_{n} \backslash I_{\text {out }}\right) \backslash\{i\} \\ \alpha_{k}\left(\theta_{k}^{\mp k}\right)<\alpha_{k}\left(x_{k}\right)<\alpha_{j}\left(x_{j}\right)^{\frac{\lambda_{k}}{\lambda_{j}}}, & k \in I_{\text {out }} \backslash\{i, j\} \\ \alpha_{j}\left(x_{j}\right)<1 . & \end{cases}
$$

With the inequalities subject to additional conditions :

- If $D_{j j} \neq \varnothing, \alpha_{j}\left(\theta_{j}^{\mp j}\right)<\alpha_{j}\left(x_{j}\right)$ has to be added to the system above.

- If $i \in I_{\text {out }}$ and $D_{i i} \neq \varnothing, \alpha_{i}\left(\theta_{i}^{\mp i}\right)^{\frac{1}{\lambda_{i}}}<\alpha_{j}\left(x_{j}\right)^{\frac{1}{\lambda_{j}}}$, has to be added.

The exponent $\varepsilon$ stands for $\mp_{i}$ if $i \in I_{\text {out }}$, and $\varepsilon \in\{+,-\}$ otherwise.

Proof. The equality satisfied by $x_{i}$ ensures that $D_{i j}^{\varepsilon} \subset W_{i}^{\varepsilon}$.

In all cases, the variables $x_{k}$, for $k \notin I_{\text {out }}$, do not influence the exit time. Hence, they are only submitted to the inequalities $\theta_{k}^{-}<x_{k}<\theta_{k}^{+}$. Reciprocally, these 
inequalities must be satisfied to ensure $x \in B$. Of course, when $i \notin I_{\text {out }}, x_{i}$ does not appear in these inequalities.

Variables $x_{k}$, for $k \in I_{\text {out }}$, must on the other hand satisfy two kinds of constraints. Firstly, they have to be between thresholds $\theta_{k}^{-}$and $\theta_{k}^{+}$. Since the functions $\alpha_{k}$ are continuous and monotone with domain $\left[\theta_{k}^{-}, \theta_{k}^{+}\right]$and range $\left[\alpha_{k}\left(\theta_{k}^{\mp k}\right), 1\right]$, for all $k \in I_{\text {out }}$, these threshold inequalities can equivalently be written

$$
\forall k \in I_{\text {out }} \backslash\{i\}, \quad \alpha_{k}\left(\theta_{k}^{\mp k}\right)<\alpha_{k}\left(x_{k}\right)<\alpha_{k}\left(\theta_{k}^{ \pm_{k}}\right)=1 .
$$

Secondly, for $k \neq j$, the corresponding exit time $\tau_{k}\left(x_{k}\right)$ must be higher than $\tau_{j}\left(x_{j}\right)$, since $D_{i j}^{\varepsilon}$ is part of $\mathcal{M}^{-1}\left(W_{j}^{ \pm_{j}}\right)$. Recall that exit times are defined in equation (5) as $\tau_{k}\left(x_{k}\right)=-\frac{1}{\lambda_{k}} \ln \left(\alpha_{k}\left(x_{k}\right)\right)$. Thus, $\tau_{k}\left(x_{k}\right)>\tau_{j}\left(x_{j}\right)$ leads to

$$
\forall k \in I_{\text {out }} \backslash\{j\}, \quad \alpha_{k}\left(x_{k}\right)<\alpha_{j}\left(x_{j}\right)^{\frac{\lambda_{k}}{\lambda_{j}}} .
$$

From $\alpha_{j}\left(x_{j}\right)<1$, and the above, we deduce $\alpha_{k}\left(x_{k}\right)<1$. This latter is hence removable from (18), for all $x_{k}, k \in I_{\text {out }} \backslash\{j\}$, but must be maintained for $x_{j}$.

For $k \in I_{\text {out }}$, and $k \neq i, j$, the inequality relating $x_{k}$ and the threshold $\theta_{k}^{\mp k}$ admits on the other hand no concurrent inequality from those of the form (19). Thus $\alpha_{k}\left(\theta_{k}^{\mp k}\right)<\alpha_{k}\left(x_{k}\right)$ has to be maintained for all $k \in I_{\text {out }} \backslash\{i\}$.

Now the last inequality we need to discuss is the one that gives a lower bound for $\alpha_{j}\left(x_{j}\right)$. To achieve this, one has to recall from the proof of lemma 1 that $D_{k k} \neq \varnothing$ admits

$$
\forall k^{\prime} \in I_{\text {out }} \backslash\{k\}, \quad \alpha_{k}\left(\theta_{k}^{\mp k}\right)^{\frac{1}{\lambda_{k}}}>\alpha_{k^{\prime}}\left(\theta_{k^{\prime}}^{\mp_{k^{\prime}}}\right)^{\frac{1}{\lambda_{k^{\prime}}}},
$$

as a set of necessary conditions. This lemma ensures moreover that, for almost all parameter values, there is exactly one $k$ such that $D_{k k} \neq \varnothing$. Thus in the following, we assume this fact.

We now consider two distinct cases.

- If $i \notin I_{\text {out }}$ : the $i$ th variable does not appear in inequalities (19).

If $D_{j j}=\varnothing$, there is (for almost all parameter values) a $k \in I_{\text {out }} \backslash\{j\}$ with $D_{k k} \neq \varnothing$. It follows that $\alpha_{j}\left(\theta_{j}^{\mp j}\right)^{\frac{1}{\lambda_{j}}}<\alpha_{k}\left(\theta_{k}^{\mp k}\right)^{\frac{1}{\lambda_{k}}}$, and then (18) and (19) together imply $\alpha_{j}\left(\theta_{j}^{\mp j}\right)<\alpha_{j}\left(x_{j}\right)$. This latter inequality has thus to be used only if $D_{j j} \neq \varnothing$, in which case it is unremovable.

- If $i \in I_{\text {out }}$ : from (19),

$$
\alpha_{i}\left(\theta_{i}^{\mp i}\right)^{\frac{\lambda_{j}}{\lambda_{i}}}<\alpha_{j}\left(x_{j}\right)
$$

holds. This competes with

$$
\alpha_{j}\left(\theta_{j}^{\mp j}\right)<\alpha_{j}\left(x_{j}\right),
$$

appearing from (18). Now, if $D_{k k} \neq \varnothing$ for some $k \neq i, j$, both lower bounds of $\alpha_{j}\left(x_{j}\right)$ are smaller than $\alpha_{k}\left(\theta_{k}^{\mp k}\right)^{\frac{\lambda_{j}}{\lambda_{k}}}$, due to (20). From (19) this quantity is a lower bound for $\alpha_{j}\left(x_{j}\right)^{\frac{1}{\lambda_{j}}}$, and thus (21) and (22) are both redundant. 
On the other hand, if either $D_{i i} \neq \varnothing$ or $D_{j j} \neq \varnothing$ (or both when $i=j$ ), one has an irredundant lower bound from (21) or (22), respectively.

Observe that in the special case where $\mathbf{H 3}$ holds, all inequalities in proposition 4 are affine, and thus the sets they describe are polytopes. We already knew this fact, but now the polytopes are explicitly described in terms of intersections of half-spaces.

From a direct count of the inequalities in proposition 4 , one can infer the corollary :

Proposition 5. Any set $D_{i j}^{\varepsilon}$ possesses $2(n-1)$ facets (which are here ( $\left.n-2\right)$-faces), except if $i \notin I_{\text {out }}$ or $D_{i i}=\varnothing$, and at the same time $D_{j j}=\varnothing$, in which case there are $2(n-1)-1$ facets.

These facets are obtained by replacing one inequality in the system by an equality.

The notions of $k$-face, and facet, are usually defined for polytopes. In the corollary above, they are extended to our piecewise smooth cells in a straightforward way.

From proposition 2, the same holds of course for sets $R_{i j}^{\varepsilon}$. Furthermore, we provide an explicit description of the latter, which is directly derived from that of the sets $D_{i j}^{\varepsilon}$.

Proposition 6. For fixed $i, j$, and $\varepsilon$, a set $R_{i j}^{\varepsilon}$ can be described by an irredundant list of inequalities directly obtained from those of $D_{i j}^{\varepsilon}$, using the translation rules below. We call $x$ a point in $D_{i j}^{\varepsilon}$, and y a point in $R_{i j}^{\varepsilon}$.

\begin{tabular}{lccc} 
& (in)equality in $D_{i j}^{\varepsilon}$ & $\leftrightarrow$ & (in)equality in $R_{i j}^{\varepsilon}$ \\
\hline equality & $x_{i}=\theta_{i}^{\varepsilon}$ & $\leftrightarrow$ & $y_{j}=\theta_{j}^{ \pm_{j}}$ \\
\hline$k \notin I_{\text {out }}$ & $x_{k}<\theta_{k}^{+}$ & $\leftrightarrow$ & $y_{k}-f_{k}<\beta_{i}\left(y_{i}\right)^{-\frac{\lambda_{k}}{\lambda_{i}}}\left(\theta_{k}^{+}-f_{k}\right)$ \\
& $x_{k}>\theta_{k}^{-}$ & $\leftrightarrow$ & $y_{k}-f_{k}>\beta_{i}\left(y_{i}\right)^{-\frac{\lambda_{k}}{\lambda_{i}}}\left(\theta_{k}^{-}-f_{k}\right)$ \\
\hline$k \in I_{\text {out }} \backslash\{i, j\}$ & $\alpha_{k}\left(x_{k}\right)<\alpha_{j}\left(x_{j}\right)^{\frac{\lambda_{k}}{\lambda_{j}}}$ & $\leftrightarrow$ & $\beta_{k}\left(y_{k}\right)<\beta_{k}\left(\theta_{k}^{ \pm}{ }^{ \pm}\right)$ \\
$\alpha_{k}\left(x_{k}\right)>\alpha_{k}\left(\theta_{k}^{\mp k}\right)$ & $\leftrightarrow$ & $\beta_{k}\left(y_{k}\right)>\beta_{i}\left(y_{i}\right)^{\frac{\lambda_{k}}{\lambda_{i}}}$ \\
\hline$\alpha_{j}\left(x_{j}\right)<1$ & $\leftrightarrow$ & $\beta_{i}\left(y_{i}\right)>1$ \\
\hline if $D_{j j} \neq \varnothing$ & $\alpha_{j}\left(x_{j}\right)>\alpha_{j}\left(\theta_{j}^{\mp j}\right)$ & $\leftrightarrow$ & $\beta_{i}\left(y_{i}\right)^{\frac{\lambda_{k}}{\lambda_{i}}}<\beta_{j}\left(\theta_{j}^{ \pm j}\right)$ \\
if $D_{i i} \neq \varnothing$ & $\alpha_{j}\left(x_{j}\right)>\alpha_{i}\left(\theta_{i}^{\mp i}\right)^{\frac{\lambda_{j}}{\lambda_{i}}}$ & $\leftrightarrow$ & $\beta_{i}\left(y_{i}\right)<\beta_{i}\left(\theta_{i}^{ \pm_{i}}\right)$ \\
\hline
\end{tabular}


Where the functions $\beta_{k}$ are given in equation (13), used in the definition of $\mathcal{M}^{-1}$.

Proof. Let $x \in D_{i j}^{\varepsilon}$, and $y=\mathcal{M} x \in R_{i j}^{\varepsilon}=\mathcal{M}\left(D_{i j}^{\varepsilon}\right)$.

The hyperplane equalities $x_{i}=\theta_{i}^{\varepsilon}$ and $y_{j}=\theta_{j}^{ \pm_{j}}$ arise directly from the definition of $D_{i j}^{\varepsilon}$ and $R_{i j}^{\varepsilon}$.

Now, from equation (13) and the definition of $\mathcal{M}^{-1}$, the two following identities are easily derived :

$$
\forall k \in \mathbb{N}_{n}, \quad\left\{\begin{array}{l}
x_{k}=f_{k}+\beta_{i}\left(y_{i}\right)^{\frac{\lambda_{k}}{\lambda_{i}}}\left(y_{k}-f_{k}\right), \\
y_{k}=f_{k}+\alpha_{j}\left(x_{j}\right)^{\frac{\lambda_{k}}{\lambda_{i}}}\left(x_{k}-f_{k}\right) .
\end{array}\right.
$$

From these one obtains :

$$
\forall k \in \mathbb{N}_{n}, \quad \alpha_{j}\left(x_{j}\right)^{\frac{\lambda_{k}}{\lambda_{j}}}=\beta_{i}\left(y_{i}\right)^{-\frac{\lambda_{k}}{\lambda_{i}}} .
$$

The functions $\beta_{k}$ are defined in equation (13) for all $k \in \mathbb{N}_{n}$.

As equations (23) show that $\theta_{k}^{-}<x_{k}<\theta_{k}^{+}$is equivalent to

$$
\theta_{k}^{+}-f_{k}>\beta_{i}\left(y_{i}\right)^{\frac{\lambda_{k}}{\lambda_{i}}}\left(y_{k}-f_{k}\right)>\theta_{k}^{-}-f_{k},
$$

the case $k \notin I_{\text {out }}$ is proved.

All other rules concern $k \in I_{\text {out }}$. Using the notation $\mp_{k}$ introduced at the beginning of this section leads to : $\beta_{k}\left(y_{k}\right)=\frac{\theta_{k}^{\mp k}-f_{k}}{y_{k}-f_{k}}$. Then all these rules are obtained using equations (23) and (24), from which simple calculations show that expressions on each side of a symbol $\leftrightarrow$ are equivalent. The last two rules are special cases of those above them, but they have been written apart since they do not always apply.

The proposition 5 can be checked on figures 3 and 4 . In these figures, one has $D_{22} \neq \varnothing$. All $D_{i j}^{\varepsilon}$ have 4 facets (here edges since they are 2-dimensional), with the exception of those with no 2 among their two indices. Namely, $D_{13}^{-}$and $D_{13}^{+}$have three edges. Of course, the same holds with the sets $R_{i j}^{\varepsilon}$.

Remarkably, these figures are only a particular case, and constructing an example with all possible instances of $D_{i j}^{\varepsilon}$ sets, in terms of facet numbers, would require more than 3 dimensions. Actually, it should contain a nonempty $D_{i i}$, with $2(n-1)$ facets, as well as some $D_{j k}, j, k \in I_{\text {out }} \backslash\{i\}$, with $2(n-1)-1$ facets. This requires 3 escaping directions, and corresponds to the example of figures 5 and 6 . Thus, to have at least one non-escaping direction $m$, such that both $D_{m i}^{ \pm}$have $2(n-1)$ facets and $D_{m j}^{ \pm}, D_{m k}^{ \pm}$have only $2(n-1)-1$, one needs a fourth dimension. This justifies the algebraic description given in propositions 4 and 6. Actually, proposition 5 gives a criterion to distinguish among configurations that are not equivalent from a combinatorial point of view. The discussion above proves that some of these configurations only occur in higher-dimensional spaces, where visualization is out of reach.

More than the usefulness of an algebraic formulation, this shows that even when dealing with a single box, the admissible behaviours form a nontrivial set. The relevance of this section is hence justified a posteriori. 


\subsection{Extension to the whole state space}

The preceding sections allow a rigorous definition of the transition mapping as an homeomorphism, at the scale of a single box, provided this latter admits escaping directions. We quickly omitted its superscript, but this local map was initially noted $\mathcal{M}^{a}: \partial B_{a}^{i n} \rightarrow \partial B_{a}^{\text {out }}$. We now provide a precise definition of the transition map on the whole state space. Although local maps are invertible on boxes with nonempty outgoing domain, boxes with no escaping direction are on the other hand more problematic. As we will see in this section, it is natural to map the boundary of such boxes to a single point, whose preimage will then be the whole box boundary. Moreover, the whole domain boundary $\partial \mathcal{U}$ is not reachable, due to H1. Hence a global mapping will not be invertible at all points in general, which leads us to consider only forward iterates of $\mathcal{M}$ in this section.

This application has to be iterated on $\bigcup_{a} \partial B_{a}$, which can naturally be seen as the underlying set of a cubical complex (see e.g. [41] for the definition of a polyhedral complex, and replace polyhedras by $n$-rectangles), whose elements are faces of the form $F=\prod_{i=1}^{n} F_{i}$ where each $F_{i}$ is of one of the following forms : $\left\{\theta_{i, a_{i}}\right\},\left\{\theta_{i, a_{i}+1}\right\}$, or $\left[\theta_{i, a_{i}}, \theta_{i, a_{i}+1}\right]$. These $F$ will be called faces, or threshold (affine) subspaces in the following. The dimension of such a face is the number of $F_{i}$ 's that are not singletons.

$\mathbf{H} 2$ implies that any outgoing facet $W \subset \partial B_{b}^{\text {out }}$, for some $b$, is part of $\partial B_{a}^{\text {in }}$, for $B_{a}$ adjacent to $B_{b}$ at wall $W$. Actually, $W \subset \partial B_{a}^{\text {out }}$ would contradict the hypothesis, and the outgoing and incoming regions of a box form a cover of its boundary. Another possibility would be that $W$ is the facet of no other box than $B_{b}$, when it lies on the boundary of the whole domain $\mathcal{U}$. But $\mathbf{H 1}$ implies that in this case $W \subset \partial B_{b}^{i n}$. Thus we get :

$$
\bigcup_{a \in \mathcal{A}} \partial B_{a}=\bigcup_{a \in \mathcal{A}} \partial B_{a}^{i n}
$$

Then any point on $\bigcup_{a \in \mathcal{A}} \partial B_{a}$ belongs to $\partial B_{a}^{\text {in }}$, for some $a \in \mathcal{A}$. If $\partial B_{a}^{\text {out }} \neq \varnothing$, then $\mathcal{M}^{a}$ is well defined, but this escaping region may also be empty, which we recall corresponds to $f(a)$ being an asymptotically stable steady state lying $\operatorname{in} \operatorname{int}\left(B_{a}\right)$. In such a case, all points in $\partial B_{a}$ are in the basin of $f(a)$, so that it seems reasonable to define $\mathcal{M}^{a}$ as the constant map with image $f(a)$. Then $\{f(a)\}$ has to be added to the domain of $\mathcal{M}^{a}$. These focal points being (asymptotically stable) equilibria, we put $\mathcal{M}^{a} f(a)=f(a)$.

After introducing the subset of terminal subscripts :

$$
\mathcal{T} \doteq\left\{a \in \mathcal{A} \mid f(a) \in \operatorname{int}\left(B_{a}\right)\right\}=\{a \in \mathcal{A} \mid \mathbf{d}(f(a))=a\},
$$

one can define local transition maps in all boxes :

$$
\mathcal{M}^{a}: x \in \operatorname{Dom}\left(\mathcal{M}^{a}\right) \longmapsto \begin{cases}f(a)+A(x)(x-f(a)) & \text { if } a \in \mathcal{A} \backslash \mathcal{T} \\ f(a) & \text { if } a \in \mathcal{T}\end{cases}
$$


where the first case is exactly identical to equation (8). The domain $\operatorname{Dom}\left(\mathcal{M}^{a}\right)=$ $\partial B_{a}^{i n}$ for $a \in \mathcal{A} \backslash \mathcal{T}$, and $\operatorname{Dom}\left(\mathcal{M}^{a}\right)=\partial B_{a}^{i n} \cup\{f(a)\}$ for $a \in \mathcal{T}$. Thus,

$$
\bigcup_{a \in \mathcal{A}} \operatorname{Dom}\left(\mathcal{M}^{a}\right)=\bigcup_{a \in \mathcal{A}} \partial B_{a} \cup \bigcup_{a \in \mathcal{T}}\{f(a)\} .
$$

Yet, a global mapping can not be properly defined. Actually, if any $x \in \bigcup_{a \in \mathcal{A}}$ $\operatorname{Dom}\left(\mathcal{M}^{a}\right)$ lies in the domain of some local map $\mathcal{M}^{a}$, the choice of this local map is not always unique. Ambiguities may happen at some face of codimension 2 (in $\mathbb{R}^{n}$ ) or more, since they lie at the intersection of at least two domains of the form above. Some of those lower dimensional faces may however lead to a well-defined flow, as seen on a simple example. Consider a binary system in the plane, with all focal points lying in the same box. Such a planar system has four boxes, indexed by $\mathbb{N}_{2} \times \mathbb{N}_{2}=\{11,12,21,22\}$. Let for example the focal points lie in $B_{22}$ : the single point with coordinates $\left(\theta_{11}, \theta_{21}\right)$ is a face with codimension 2 , on which all neighbouring flow lines are directed towards $B_{22}$. Hence the flow is not ambiguous on this face, and leads to the asymptotically stable steady state $f(22)$.

Other, less simple, examples also exhibit lower dimensional faces with a welldefined flow. A formal characterization of the faces on which the flow is ambiguous is not presently known. We believe that some combinatorial criterion, involving the positions of all neighbouring focal points with respect to neighbouring boxes, might do the trick. Since such a criterion is still lacking, and in order to simplify the analysis, we chose here to exclude all codimension 2 faces from the analysis, along with the subset from which those faces can be reached. On such a domain, a global map can finally be well defined, and written for example with the aid of characteristic functions $\left(\mathbf{1}_{A}(x)=1\right.$ for $x \in A, 0$ otherwise) :

$$
\mathcal{M} x=\sum_{a \in \mathcal{A}} \mathbf{1}_{\operatorname{Dom}\left(\mathcal{M}^{a}\right)}(x) \mathcal{M}^{a} x .
$$

And letting $\mathscr{F}_{2}$ be the union of all threshold faces of codimension 2 or more, the domain $\mathscr{D}$ on which $\mathcal{M}$ and its iterates are well defined may be written :

$$
\mathscr{D}=\bigcup_{a \in \mathcal{A}} \operatorname{Dom}\left(\mathcal{M}^{a}\right) \backslash \bigcup_{k \in \mathbb{N}} \mathcal{M}^{-k}\left(\mathscr{F}_{2}\right)
$$

The notation $\mathcal{M}^{k}$ stands for the $k$ th iterate of $\mathcal{M}$ defined in (27), and $\mathcal{M}^{-k}\left(\mathscr{F}_{2}\right)$ is then the preimage of the set $\mathscr{F}_{2}$. This requires implicitly that previous iterates are well defined, i.e. belong to $\mathscr{D}$ as well. The excluded set is thus the union of all finite time preimages of codimension 2 faces. As such, its measure is zero in $\operatorname{Dom}\left(\mathcal{M}^{a}\right)$, and the restriction is not too strong.

Remark 3. In the paragraph above, the term "finite time" is to be understood as "finite number of iterates". This distinction must be considered carefully, since Edwards [10] has shown that some trajectories can cross an infinite sequence of boxes in finite time. He showed that such trajectories always converge towards a face in the set we call $\mathscr{F}_{2}$. 
On the other hand, the domain $\mathscr{D}$ is not closed in $\bigcup_{a} \operatorname{Dom}\left(\mathcal{M}^{a}\right)$, and thus it is not compact. Actually, a sequence in $\mathscr{D}$ that converges to a point in some codimension 2 face is easily contructed. In particular, some orbits in $\mathscr{D}$ may have an $\omega$-limit set that does not belong to $\mathscr{D}$. Typical examples of such orbits are those converging towards a stable focus lying at the intersection of two walls or more, without intersecting any such lower dimensional face in finite time (i.e. a finite number of iterations).

Remark that $\mathscr{D}$ is not open either, since in general its complement $\bigcup_{k} \mathcal{M}^{-k}\left(\mathscr{F}_{2}\right)$ is not closed. This arises from the fact that the latter is an infinite union of closed sets. As there are only finitely many faces in $\mathcal{M}$ 's domain, the only possibility for the previous union to be really infinite, and to preclude closedness, is the existence of a periodic or quasiperiodic unstable orbit $\mathcal{O}$. Then there is a neighbourhood $\mathcal{N}=\mathcal{N}(\mathcal{O})$ such that, for any $x \in \mathcal{N}$, the trajectory $\left(\mathcal{M}^{k} x\right)_{k}$ escapes from $\mathcal{N}$ as $k \rightarrow+\infty$, and admits $\mathcal{O}$ as $\alpha$-limit set, i.e. as set of limit points when $k \rightarrow-\infty$. Since the solutions of the affine differential equations in each box are continuous with respect to the initial condition, there exist some initial points $x \in \mathcal{N}$ whose trajectory intersect $\mathscr{F}_{2}$, as soon as one box around $\mathcal{N}$ admits two successors or more. Then, on such a trajectory, all points lie in the complement of $\mathscr{D}$. On the other hand, the sequence $\left(\mathcal{M}^{-k} x\right)_{k}$ admits $\mathcal{O}$ as a limit set, and $\mathcal{O} \subset \mathscr{D}$. Hence, some subsequence of $\left(\mathcal{M}^{-k} x\right)_{k}$ entirely lies in the complement of $\mathscr{D}$, and converges towards some limit point in $\mathscr{D}$. It follows that the complement is not closed, and thus $\mathscr{D}$ is not open. We do not provide an explicit example for such a configuration here, but it is realizable. Actually, unstable limit cycles are also realizable orbits, as will appear more explicitly in proposition 10. More concretely, the strange attractor presented in [33] explicitly provides an example of unstable limit cycle. In the absence of configuration of the form described above, $\mathscr{D}$ is an open set. We plan to provide such an example in future work.

A last observation is that $\mathscr{D}$ is not a connected space. This is clear from the fact that $\mathscr{D}$ is a subset of all walls in phase space, taken without their boundaries. Since these open walls are disjoint, and $\mathscr{D}$ clearly has nonempty intersection with all of them, it can not be connected. The connected components of $\mathscr{D}$ will be made explicit in section 5 .

Despite its somehow clumsy topology, the set $\mathscr{D}$ is almost (i.e. up to a zeromeasure set) the largest one on which all iterates of $\mathcal{M}$ are well defined. The true largest domain on which a global mapping is well-defined, along with all its iterates, is not simple at all, as discussed below eq. (26). Moreover, $\mathcal{M}$ is continuous on the domain $\mathscr{D}$, since it is essentially $\bigcup_{a} \operatorname{Dom}\left(\mathcal{M}^{a}\right)$ with all discontinuity points removed.

Now, $(\mathscr{D}, \mathcal{M})$ is a properly defined one-sided discrete dynamical system. The orbits in this system are of the form $\left\{\mathcal{M}^{k} x\right\}_{k \in \mathbb{N}}$, for some $x \in \mathscr{D}$. The iterates of $\mathcal{M}$ are in fact compositions of local maps, which depend on the sequence of walls that are crossed by the orbits. The next section is devoted to the analysis of such iterates, in particular along cyclic sequences of walls. 


\section{Composite maps}

We call cycle maps the $k$ th iterate of $\mathcal{M}$ along a cyclic sequence of $k$ walls, seen as a first return map, defined on a subset of a wall. We deal in this section under assumption $\mathbf{H 3}$, since otherwise computations rapidly become intractable, and the linear algebra tools we use here cannot be invoked.

The task of describing cycle maps' domains and fixed points has been investigated in early studies on systems of the form (1), mainly [21,22,32]. All these previous results, as well as some new ones can be found in a work of R. Edwards [10], with recent improvements given in [13] in terms of combining multiple loops. These studies all concern the case of a single threshold per direction, which is translated to zero : then, $\mathcal{M}$ is a fractional linear mapping, i.e. a linear mapping divided by an affine 1-form. Composition preserves such mappings. Given a cyclic sequence of boxes, it is shown that the domain on which a return map is well defined is a polyhedral cone. Furthermore, fixed points of the return maps are closely related to eigenvectors of the linear numerator of the return map. Although it is commonly thought that these results extend to the case of multiple thresholds, this has not been properly proved yet.

Here, we propose such an extension to the multiple thresholds context. This leads to dealing with fractional affine mappings instead of fractional linear ones. In short, the main difference with previous results is that one has to consider translations of eigenspaces, instead of eigenspaces themselves, and keep track of all crossed thresholds along an orbit, since they are not all the same. This not only complicates the formulae and computations, but one also loses some nice features of the binary case, as discussed at the end of this section.

Recall that since $\mathbf{H 3}$ is assumed, in a box $B_{a}$ the mapping $\mathcal{M}=\mathcal{M}^{a}$ can be written :

$$
\mathcal{M} x=f(a)+\alpha(x)(x-f(a))=f(a)+\frac{\theta_{\iota}^{ \pm_{\iota}}-f_{\iota}}{x_{\iota}-f_{\iota}}(x-f(a)),
$$

where $\iota$ is the escaping direction for $x$, and $\theta_{\iota}^{ \pm_{\iota}}$ is either $\theta_{\iota}^{-} \doteq \theta_{\iota a_{\iota}}$ or $\theta_{\iota}^{+} \doteq \theta_{\iota a_{\iota}+1}$ (the choice being given by the condition $\alpha(x) \in] 0,1[$, for example).

\subsection{Iterates of the transition map}

A matrix formulation of $\mathcal{M}$ 's iterates can be obtained from the equation above. Given a wall $W, \ell$ successive iterates of the transition map can follow different sequences of facets. Accordingly, $W$ can be partitioned into regions corresponding to specific wall sequences. In particular, if there is a periodic sequence of $\ell$ crossed boxes, containing $W$, one of these domains correspond to the corresponding loop. A necessary condition for a limit cycle to happen is then naturally that this domain be nonempty.

More explicitly, let $\mathbf{a}=a^{0} \ldots a^{k+1}$ be a finite sequence of symbols in $\mathcal{A}$, such that there may be some continuous trajectory intersecting successively $B_{a^{0}} \ldots B_{a^{k+1}}$. It follows that such a trajectory crosses successively the walls $W^{0} \doteq \partial B_{a^{0}}^{\text {out }} \cap \partial B_{a^{1}}^{\text {in }}$, 
$\ldots W^{k} \doteq \partial B_{a^{k}}^{\text {out }} \cap \partial B_{a^{k+1}}^{i n}$. Then, on the wall $W^{0} \subset \partial B_{a^{1}}^{i n}$, the domain from which the sequence $\mathbf{a}$ is followed can be written as :

$$
D_{\mathbf{a}} \doteq \bigcap_{i=0}^{k} \mathcal{M}^{-i}\left(W^{i}\right)
$$

This expression is reminiscent of the definition of the domains $D_{i j}^{\varepsilon}(a)$, in equation (14). In fact, the latter correspond to the particular case $k=1$ in the formula above, with appropriate a. Note that the word a can not be arbitrary, and that the set $D_{\mathbf{a}}$ may be empty. This will be analysed in more detail in section 5 .

Within the framework of binary systems, sets of the form (30) are easily defined as polyhedral cones. Moreover, the linear inequalitites defining these cones are obtained via a simple rescaling of the linear part of the transition map. Since boxes are orthants in this context, describing a set of the form $D_{\mathbf{a}}$ consists in forcing the sign pattern of solutions of the linear inequalities describing the returning cone. In our more general context, an explicit description of domains $D_{\mathbf{a}}$ is still possible, though in less simple form. We postpone this description until the end of the section, after describing iterates of $\mathcal{M}$ on a fixed sequence of walls.

As focal points lying inside their own originating box are a special case, for which $\mathcal{M}$ has been defined as a constant map with no change in forward iterates, we assume in the following that all crossed boxes have at least one escaping direction.

On a domain $D_{\mathbf{a}}$ (supposed nonempty until further clarifications), corresponding to a fixed list of $k$ walls successively crossed by the flow, $\mathcal{M}^{k}$ can be written unambiguously. Observe that since $W^{0}=\partial B_{a^{0}}^{\text {out }} \cap \partial B_{a^{1}}^{\text {in }}$, the symbol of the first box crossed by orbits originating in $D_{\mathbf{a}}$ is $a^{1}$, and not $a^{0}$. Let accordingly $f^{1} \ldots f^{k}$ be the focal points of the successively crossed boxes (i.e. $f^{j}=f\left(a^{j}\right)$ ), and $\iota_{1}, \ldots, \iota_{k}$ the exit directions followed along these boxes. This leads to the following expression :

$$
\forall x \in D_{\mathbf{a}}, \quad \mathcal{M}^{k} x=f^{k}+\Delta_{\iota_{k}} \frac{F^{(k)}\left(x-f^{1}\right)}{\left\langle F^{(k)}\left(x-f^{1}\right), \mathbf{e}_{\iota_{k}}\right\rangle},
$$

where the term $\Delta_{\iota_{k}}$ above is an abbreviation for $\theta_{l_{k}}^{ \pm_{l_{k}}}-f_{l_{k}}^{k}$, with the threshold $\theta_{\iota_{k}}^{ \pm} \pm_{\iota_{k}} \in\left\{\theta_{\iota_{k} a_{\iota_{k}}}, \theta_{\iota_{k} a_{\iota_{k}}+1}\right\}$ being the $k$ th to be crossed. From H1, $\Delta_{\iota_{k}}$ is clearly nonzero. Noting transposition with a ${ }^{T},\langle x, y\rangle=x^{T} y=y^{T} x$ is the usual inner product, introduced here to improve readability.

The matrix $F^{(k)} \in \mathbb{R}^{n \times n}$ is defined as the right-to-left product :

$$
\begin{aligned}
F^{(k)} & =\prod_{j=1}^{k-1}\left[\left(f^{j}-f^{j+1}\right) \mathbf{e}_{\iota_{j}}^{T}+\Delta_{\iota_{j}} I d\right] \\
& =\left[\left(f^{k-1}-f^{k}\right) \mathbf{e}_{\iota_{k-1}}^{T}+\Delta_{\iota_{k-1}} I d\right] \ldots\left[\left(f^{1}-f^{2}\right) \mathbf{e}_{\iota_{1}}^{T}+\Delta_{\iota_{1}} I d\right],
\end{aligned}
$$

for $k \geqslant 2$, and $F^{(1)}=I d$. 
The expression of $\mathcal{M}^{k}$ is easily proved by induction. Actually, supposing it correct at a step $k$, and computing $\mathcal{M}^{k+1}$ on a domain where the escaping direction is $\iota_{k+1}$, one gets from (29), which also gives the initial step of the induction :

$$
\mathcal{M}^{k+1} x=f^{k+1}+\frac{\Delta_{\iota_{k+1}}}{\left(\mathcal{M}^{k} x\right)_{\iota_{k+1}}-f_{\iota_{k+1}}^{k+1}}\left(\mathcal{M}^{k} x-f^{k+1}\right),
$$

and reinjecting eq. (31), i.e. the induction hypothesis,

$$
\begin{aligned}
\mathcal{M}^{k+1} x & =f^{k+1}+\Delta_{\iota_{k+1}} \frac{f^{k}+\Delta_{\iota_{k}} \frac{F^{(k)}\left(x-f^{1}\right)}{\left\langle F^{(k)}\left(x-f^{1}\right), \mathbf{e}_{\iota_{k}}\right\rangle}-f^{k+1}}{\left\langle f^{k}+\Delta_{\iota_{k}} \frac{F^{(k)}\left(x-f^{1}\right)}{\left\langle F^{(k)}\left(x-f^{1}\right), \mathbf{e}_{\iota_{k}}\right\rangle}-f^{k+1}, \mathbf{e}_{\iota_{k+1}}\right\rangle} \\
& =f^{k+1}+\Delta_{\iota_{k+1}} \frac{\left(f^{k}-f^{k+1}\right) \mathbf{e}_{\iota_{k}}^{T} F^{(k)}\left(x-f^{1}\right)+\Delta_{\iota_{k}} F^{(k)}\left(x-f^{1}\right)}{\left\langle\left(f^{k}-f^{k+1}\right) \mathbf{e}_{\iota_{k}}^{T} F^{(k)}\left(x-f^{1}\right)+\Delta_{\iota_{k}} F^{(k)}\left(x-f^{1}\right), \mathbf{e}_{\iota_{k+1}}\right\rangle} \\
& =f^{k+1}+\Delta_{\iota_{k+1}} \frac{F^{(k+1)}\left(x-f^{1}\right)}{\left\langle F^{(k+1)}\left(x-f^{1}\right), \mathbf{e}_{\iota_{k+1}}\right\rangle}
\end{aligned}
$$

with $F^{(k+1)}=\left[\left(f^{k}-f^{k+1}\right) \mathbf{e}_{\iota_{k}}^{T}+\Delta_{\iota_{k}} I d\right] F^{(k)}$, yielding (32).

This matrix is always invertible, as we show now.

Proposition 7. For all $k \in \mathbb{N}$, the $n \times n$ matrix $F^{(k)}$ is invertible, as far as $\mathbf{H 1}$ holds.

Proof. From equation (32), $F^{(k)}$ is a product of $n \times n$ matrices, which are all diagonal except for one nonzero column. Developing along this column, only one minor is nonzero, yielding for the $j$ th matrix :

$$
\begin{aligned}
\operatorname{det}\left(\left(f^{j}-f^{j+1}\right) \mathbf{e}_{\iota_{j}}^{T}+\Delta_{\iota_{j}} I d\right) & =\left(\Delta_{\iota_{j}}\right)^{n-1}\left(\Delta_{\iota_{j}}+f_{\iota_{j}}^{j}-f_{\iota_{j}}^{j+1}\right) \\
& =\left(\theta_{\iota_{j}}^{ \pm_{\iota_{j}}}-f_{\iota_{j}}^{j}\right)^{n-1}\left(\theta_{\iota_{j}}^{ \pm_{\iota_{j}}}-f_{\iota_{j}}^{j+1}\right),
\end{aligned}
$$

whence the determinant :

$$
\operatorname{det}\left(F^{(k)}\right)=\prod_{j=1}^{k-1}\left(\Delta_{\iota_{j}}\right)^{n-1}\left(\theta_{\iota_{j}}^{ \pm_{\iota_{j}}}-f_{\iota_{j}}^{j+1}\right),
$$

which from assumption $\mathbf{H 1}$ is nonzero, whatever $k$.

It is implicitly assumed, when writing equation (31) for $\mathcal{M}^{k}$, that the inner product $\left\langle F^{(k)}\left(x-f^{1}\right), \mathbf{e}_{\iota_{k}}\right\rangle \neq 0$ in the denominator. As can be seen from an alternative way of expressing $\mathcal{M}^{k}$, this condition is always verified. This relies on Edwards observation, in [10], that the time spent on the continuous trajectory between some $x$ and its image $\mathcal{M}^{k} x$ can be read directly from $\mathcal{M}^{k}$, in the case of binary systems. This property is still valid in our case. Letting $\tau^{j}(x)=\tau\left(\mathcal{M}^{j-1} x\right)$ be the time spent in the $j$ th crossed box, for $j=1 \ldots k$, the following holds :

$$
\exp \left(-\lambda \sum_{j=1}^{k} \tau^{j}(x)\right)=\frac{\Delta_{\iota_{1}} \ldots \Delta_{\iota_{k}}}{\left\langle F^{(k)}\left(x-f^{1}\right), \mathbf{e}_{\iota_{k}}\right\rangle},
$$


where the sum in the exponential is clearly the total time between $x$ and $\mathcal{M}^{k} x$. Recall that since $\mathbf{H 3}$ is assumed, the degradation rate $\lambda$ does not need a subscript. A zero inner product would mean that this time is infinite (and negative !). Such a pathological case is only possible along a finite sequence of boxes, one of which contains its own focal point. But the latter case has been excluded here, as explained before.

To prove that equation (34) holds, one has to remember that $\alpha(x)=e^{-\lambda \tau(x)}$, in equation (29). Then, for an iterate of $\mathcal{M}$ there are two equivalent formulations :

$$
\mathcal{M}^{j} x-f^{j}=e^{-\lambda \tau^{j}(x)}\left(\mathcal{M}^{j-1} x-f^{j}\right)=\Delta_{\iota_{j}} \frac{F^{(j)}\left(x-f^{1}\right)}{\left\langle F^{(j)}\left(x-f^{1}\right), \mathbf{e}_{\iota_{j}}\right\rangle} .
$$

Projecting this expression on $\mathbf{e}_{\iota_{j}}$, the scalar $e^{-\lambda \tau^{j}(x)}$ can be expressed as a ratio :

$$
e^{-\lambda \tau^{j}(x)}=\frac{\Delta_{\iota_{j}}}{\left\langle\mathcal{M}^{j-1} x-f^{j}, \mathbf{e}_{\iota_{j}}\right\rangle}
$$

From equations (31) and (32) :

$$
\begin{aligned}
\mathcal{M}^{j-1} x-f^{j} & =\frac{\left[\left(f^{j-1}-f^{j}\right) \mathbf{e}_{\iota_{j-1}}^{T}+\Delta_{j-1} I d\right] F^{(j-1)}\left(x-f^{1}\right)}{\left\langle F^{(j-1)}\left(x-f^{1}\right), \mathbf{e}_{\iota_{j-1}}\right\rangle} \\
& =\frac{F^{(j)}\left(x-f^{1}\right)}{\left\langle F^{(j-1)}\left(x-f^{1}\right), \mathbf{e}_{\iota j-1}\right\rangle},
\end{aligned}
$$

which leads to

$$
e^{-\lambda \tau^{j}(x)}=\frac{\Delta_{\iota_{j}}\left\langle F^{(j-1)}\left(x-f^{1}\right), \mathbf{e}_{\iota_{j-1}}\right\rangle}{\left\langle F^{(j)}\left(x-f^{1}\right), \mathbf{e}_{\iota_{j}}\right\rangle} .
$$

Equation (34) is then a product of ratios of the form above.

Hence, one gets an alternative formulation of equation (31) in which the time is explicit :

$$
\mathcal{M}^{k} x=f^{k}+\frac{\exp \left(-\lambda \sum_{j=1}^{k} \tau^{j}(x)\right)}{\Delta_{\iota_{1}} \ldots \Delta_{\iota_{k-1}}} F^{(k)}\left(x-f^{1}\right) .
$$

Now we have all the ingredients to provide an explicit description of the domains $D_{\mathbf{a}}$, eq. (30). Inequalities defining this domain are of two kinds : those expressing the inclusion $D_{\mathbf{a}} \subset W^{0}$, and those expressing that orbits initially follow the sequence of boxes a. The first are immediate, and the difficulty only comes from the second. Observe that we will not give an irredundant description, as we were able to do in the simple case of proposition 4, even if we deal here under the simplifying assumption H3. 
Proposition 8. For $\mathbf{a}=a^{0} \ldots a^{k+1}$, the set $D_{\mathbf{a}}$, as defined in equation (30) is properly described by the following set of inequalities, for each $j \in \mathbb{N}_{k}$ :

$$
\forall i \in I_{\text {out }}\left(a^{j}\right) \backslash\left\{\iota_{j}\right\}, \quad \frac{1}{\Delta_{i} \Delta_{\iota_{1}} \ldots \Delta_{\iota_{j}}}\left[\Delta_{\iota_{j}} \mathbf{e}_{i}^{T}-\Delta_{i} \mathbf{e}_{\iota_{j}}^{T}\right] F^{(j)}\left(x-f^{1}\right)>0,
$$

along with the $2(n-1)$ inequalities defining $W^{0} \supset D_{\mathbf{a}}$.

The value $\theta_{i}^{ \pm_{i}}$ is the escaping threshold in direction $i$ for the box $B_{a^{j}}, \Delta_{i}$ is similar to the already defined abbreviation of $\theta_{i}^{ \pm_{i}}-f_{i}^{j}$. Since for each $i, x-f^{1}$ is multiplied on the left by $a 1 \times n$ vector in inequality (36), the system of inequalities associated with each $j$ can be put in matrix form as $C^{(j)}\left(x-f^{1}\right)>0$, where the rows of $C^{(j)}$ are given by (36).

Proof. The necessity of inequalities defining $W^{0}$ does not need any proof.

Other inequalities are equivalent to $\tau_{i}\left(\mathcal{M}^{j-1} x\right)>\tau_{\iota_{j}}\left(\mathcal{M}^{j-1} x\right)$, i.e. $\iota_{j}$ is the escaping direction in $B_{a^{j}}$, for initial condition $\mathcal{M}^{j-1} x$.

Actually, denoting $x^{j} \doteq \mathcal{M}^{j} x$ for concision, the later inequality on exit times can be rewritten :

$$
\frac{x_{i}^{j-1}-f_{i}^{j}}{\Delta_{i}}-\frac{x_{\iota_{j}}^{j-1}-f_{\iota_{j}}^{j}}{\Delta_{\iota_{j}}}>0 .
$$

This has clearly to be statisfied by all $i \in I_{\text {out }}\left(a^{j}\right) \backslash\left\{\iota_{j}\right\}$, and is equivalent to :

$$
\frac{1}{\Delta_{i} \Delta_{\iota_{j}}}\left[\Delta_{\iota_{j}} \mathbf{e}_{i}^{T}-\Delta_{i} \mathbf{e}_{\iota_{j}}^{T}\right]\left(x^{j-1}-f^{j}\right)>0 .
$$

Now, from eq. (35) and $x^{j-1}-f^{j}=e^{\lambda \tau^{j}(x)}\left(x^{j}-f^{j}\right)$, one gets :

$$
x^{j-1}-f^{j}=\frac{\exp \left(-\lambda \sum_{i=1}^{j-1} \tau^{i}(x)\right)}{\Delta_{\iota_{1}} \ldots \Delta_{\iota_{j-1}}} F^{(j)}\left(x-f^{1}\right),
$$

and since the exponential is positive, inequality (36) follows.

\subsection{Cyclic maps}

Now we turn to the case of cyclic maps. For this, let $a^{0}, a^{1}, \ldots, a^{\ell}=a^{0}$ be a periodic list of box symbols, so that $D_{\mathbf{a}} \neq \varnothing$, for $\mathbf{a}=a^{0} \ldots a^{\ell-1} a^{0}$. There are then corresponding periodic sequences of walls, focal points, and exiting directions : $W^{j}, f^{j}$ and $\iota_{j}$, with $j$ taken modulo $\ell$. Then, for an $x \in D_{\mathbf{a}} \subset W^{0}$, the iterated map $\mathcal{M}^{\ell}$ maps $x$ back on $W^{0}$, and writes :

$$
\mathcal{M}^{\ell} x=f^{0}+\Delta_{\iota_{0}} \frac{F^{(\ell)}\left(x-f^{1}\right)}{\left\langle F^{(\ell)}\left(x-f^{1}\right), \mathbf{e}_{\iota_{0}}\right\rangle},
$$

where the periodicity of the box sequence leads to replacing occurences of $\ell$ by 0 above, as well as in the expression of $F^{(\ell)}$ computed from (32) (but $F^{(\ell)} \neq F^{(0)}$, of course). 
Now, for nonempty $D_{\mathbf{a}}$ the restriction $\left.\mathcal{M}^{\ell}\right|_{D_{\mathbf{a}}}$ is not strictly speaking a Poincaré return map in general, since some points may be mapped outside $D_{\mathbf{a}}$, and thus escape from the loop a when mapped again. However, it is clear that if a limit cycle exists around $\mathbf{a}$, it must correspond to a fixed point of $\mathcal{M}^{\ell}$ that lies in $D_{\mathbf{a}}$. A practical way to characterize fixed points of this map is to translate the expression (37) :

$$
\mathcal{M}^{\ell} x-f^{1}=f^{0}-f^{1}+\Delta_{\iota_{0}} \frac{F^{(\ell)}\left(x-f^{1}\right)}{\left\langle F^{(\ell)}\left(x-f^{1}\right), \mathbf{e}_{\iota_{0}}\right\rangle},
$$

which can be put as a single fractional term :

$$
\begin{aligned}
\mathcal{M}^{\ell} x-f^{1} & =\frac{1}{\left\langle F^{(\ell)}\left(x-f^{1}\right), \mathbf{e}_{\iota_{0}}\right\rangle}\left[\left(f^{0}-f^{1}\right) \mathbf{e}_{\iota_{0}}^{T}+\Delta_{\iota_{0}} I d\right] F^{(\ell)}\left(x-f^{1}\right) \\
& =\frac{F^{(\ell+1)}\left(x-f^{1}\right)}{\left\langle F^{(\ell)}\left(x-f^{1}\right), \mathbf{e}_{\iota_{0}}\right\rangle}
\end{aligned}
$$

Here again, $F^{(\ell+1)}$ is obtained from eq. (32) with indices taken modulo $\ell$.

It appears now that if $x^{*}$ is a fixed point of the return map $\mathcal{M}^{\ell}$, then $x^{*}-f^{1}$ is an eigenvector of $F^{(\ell+1)}$, with eigenvalue $\left\langle F^{(\ell)}\left(x^{*}-f^{1}\right), \mathbf{e}_{\iota_{0}}\right\rangle$.

Conversely, if $y-f^{1}$ is an eigenvector of $F^{(\ell+1)}$, with eigenvalue $\mu, y$ is a fixed point of $\mathcal{M}^{\ell}$ only if it satisfies :

$$
\mu=\left\langle F^{(\ell)}\left(y-f^{1}\right), \mathbf{e}_{\iota_{0}}\right\rangle
$$

as seen by simply combining the two requirements $F^{(\ell+1)}\left(y-f^{1}\right)=\mu\left(y-f^{1}\right)$ and $\mathcal{M}^{\ell} y=y$. Thus, writing $E_{\mu}\left(F^{(\ell+1)}\right)$, or simply $E_{\mu}$ when nonambiguous, for the eigenspace corresponding to an eigenvalue $\mu$, and given a point $y \in f^{1}+E_{\mu}$, a fixed point for $\mathcal{M}^{\ell}$ can be calculated :

$$
x^{*}=f^{1}+\frac{\mu}{\left\langle F^{(\ell)}\left(y-f^{1}\right), \mathbf{e}_{\iota_{0}}\right\rangle}\left(y-f^{1}\right),
$$

provided it belongs to $D_{\mathbf{a}}$, and $\left\langle F^{(\ell)}\left(y-f^{1}\right), \mathbf{e}_{\iota_{0}}\right\rangle \neq 0$.

This latter condition is necessary for a fixed point to belong to the line $f^{1}+$ $\mathbb{R}\left(y-f^{1}\right)$. Actually, the nonzero inner product above is invariant when $y$ is replaced by a point in the affine line $f^{1}+\mathbb{R}\left(y-f^{1}\right)$, and we have shown with eq. (34) that this inner product must be nonzero for all images of the return map, hence for fixed points.

In fact, we show now that this condition necessarily holds if $f^{1}+\mathbb{R}\left(y-f^{1}\right)$ intersects the hyperplane $\left\{x \mid x_{\iota_{0}}=\theta_{\iota_{0}}^{ \pm_{\iota_{0}}}\right\}$. In particular, it is automatically satisfied if the point $x^{*}$ in equation (39) belongs to $D_{\mathbf{a}}$, which is a subset of the wall $W^{0}$, itself included in the mentioned hyperplane.

Proposition 9. Let $\mu$ be an eigenvalue of matrix $F^{(\ell+1)}$, and $\left(y-f^{1}\right) \in E_{\mu}$ a corresponding eigenvector. Suppose $\mathbf{H 1}$, and $f^{1}+\mathbb{R}\left(y-f^{1}\right) \cap\left\{x \mid x_{\iota_{0}}=\theta_{\iota_{0}}^{ \pm_{\iota_{0}}}\right\} \neq \varnothing$. Then, $\left\langle F^{(\ell)}\left(y-f^{1}\right), \mathbf{e}_{\iota_{0}}\right\rangle \neq 0$. 
Proof. Suppose $\left\langle F^{(\ell)}\left(y-f^{1}\right), \mathbf{e}_{\iota_{0}}\right\rangle=0$. Then,

$$
\begin{aligned}
F^{(\ell+1)}\left(y-f^{1}\right) & =\left[\left(f^{0}-f^{1}\right) \mathbf{e}_{\iota_{0}}^{T}+\Delta_{\iota_{0}} I d\right] F^{(\ell)}\left(y-f^{1}\right) \\
& =\Delta_{\iota_{0}} F^{(\ell)}\left(y-f^{1}\right),
\end{aligned}
$$

so that $\left\langle F^{(\ell+1)}\left(y-f^{1}\right), \mathbf{e}_{l_{0}}\right\rangle=0$. And since $F^{(\ell+1)}\left(y-f^{1}\right)=\mu\left(y-f^{1}\right)$ with nonzero $\mu$ (due to proposition 7), this orthogonality equation yields $y_{\iota_{0}}=f_{\iota_{0}}^{1}$. Such a relation holds for any point in the affine line $f^{1}+\mathbb{R}\left(y-f^{1}\right)$. In particular, if a point of this line also belongs to the hyperplane of the proposition's statement, one is lead to the equality : $y_{\iota_{0}}=\theta_{\iota_{0}}^{ \pm_{\iota_{0}}}=f_{\iota_{0}}^{1}$. This contradicts $\mathbf{H 1}$.

We have given a necessary condition on eigenvectors, let us now give one on eigenvalues. Since this product will appear several times, an abbreviation may be introduced :

$$
\Delta \doteq \prod_{j=1}^{\ell} \Delta_{\iota_{j}} .
$$

Then the the necessary condition is :

$$
\frac{\mu}{\Delta}>1
$$

for a fixed point to be obtained from the eigenspace $E_{\mu}$. This comes directly from eq. (34), since transition times must be positive, and the inner product equals the eigenvalue associated with a candidate fixed point. Then, $\Delta$ and $\mu$ must have the same sign, and the latter must be real and of greater modulus than $\Delta$.

As a notable point, the sign of $\Delta$ is fully determined from the parity of the cycle's length $\ell$. Actually, the sequence of crossed walls is periodic. Then, if the flow crosses a wall with threshold $\theta_{\iota_{j}}^{ \pm_{\iota_{j}}}$ increasing in direction $\iota_{j}$, which implies $\Delta_{\iota_{j}}<0$, it must decrease across the same hyperplane at some later point on the same orbit, so that $\Delta_{l j+m}>0$, for some $m$. Thus, the full product consists of such pairs, whose product is negative. Hence $\ell$ must be even, and

$$
\operatorname{sign}(\Delta)=(-1)^{\frac{\ell}{2}}
$$

The case of equality in eq. (41) is to be rejected when looking for fixed points. This has to be stressed, since $\Delta$ is a potential eigenvalue of $F^{(\ell+1)}$, with any $\mathbf{e}_{i}$ as eigenvector, where $i$ is a direction in which no wall is crossed along the cycle. This results directly from eq. (32), when at least one $i \in \mathbb{N}_{n}$ does not appear as an exit direction $\iota_{j}$, thus leading to the $i$ th column being nonzero on the diagonal only, with entry $\Delta$.

Up to now, all results we have given concerning cycle maps were already known (with simpler form) in the binary case, as analyzed in [10]. In the latter work, stability of fixed points is also investigated. Here again, with slight differences, the results 
extend. To show this, we compute the Jacobian of our return map (equation (38)) at a point $x$ :

$$
D \mathcal{M}_{x}^{\ell}=\frac{F^{(\ell+1)}}{\left\langle F^{(\ell)}\left(x-f^{1}\right), \mathbf{e}_{\iota_{0}}\right\rangle}-\frac{F^{(\ell+1)}\left(x-f^{1}\right) \mathbf{e}_{\iota_{0}}^{T} F^{(\ell)}}{\left\langle F^{(\ell)}\left(x-f^{1}\right), \mathbf{e}_{\iota_{0}}\right\rangle^{2}},
$$

which, at a fixed point $x^{*}$, takes the simpler form :

$$
D \mathcal{M}_{x^{*}}^{\ell}=\frac{F^{(\ell+1)}}{\left\langle F^{(\ell)}\left(x^{*}-f^{1}\right), \mathbf{e}_{\iota_{0}}\right\rangle}-\frac{\left(x^{*}-f^{1}\right) \mathbf{e}_{l_{0}}^{T} F^{(\ell)}}{\left\langle F^{(\ell)}\left(x^{*}-f^{1}\right), \mathbf{e}_{\iota_{0}}\right\rangle} .
$$

Now we apply this Jacobian at $x^{*}-f^{1}$ :

$$
D \mathcal{M}_{x^{*}}^{\ell}\left(x^{*}-f^{1}\right)=\left(x^{*}-f^{1}\right)-\left(x^{*}-f^{1}\right)=0 .
$$

Given another fixed point $y^{*}$, one also computes the following :

$$
\begin{aligned}
D \mathcal{M}_{x^{*}}^{\ell}\left(y^{*}-x^{*}\right)= & D \mathcal{M}_{x^{*}}^{\ell}\left(y^{*}-f^{1}\right)-D \mathcal{M}_{x^{*}}^{\ell}\left(x^{*}-f^{1}\right) \\
= & \frac{F^{(\ell+1)}\left(y^{*}-f^{1}\right)}{\left\langle F^{(\ell)}\left(x^{*}-f^{1}\right), \mathbf{e}_{\iota_{0}}\right\rangle}-\frac{\left(x^{*}-f^{1}\right) \mathbf{e}_{\iota_{0}}^{T} F^{(\ell)}\left(y^{*}-f^{1}\right)}{\left\langle F^{(\ell)}\left(x^{*}-f^{1}\right), \mathbf{e}_{\iota_{0}}\right\rangle} \\
= & \frac{\left\langle F^{(\ell)}\left(y^{*}-f^{1}\right), \mathbf{e}_{\iota_{0}}\right\rangle\left(y^{*}-f^{1}\right)}{\left\langle F^{(\ell)}\left(x^{*}-f^{1}\right), \mathbf{e}_{\iota_{0}}\right\rangle} \\
& -\frac{\left(x^{*}-f^{1}\right)\left\langle F^{(\ell)}\left(y^{*}-f^{1}\right), \mathbf{e}_{\iota_{0}}\right\rangle}{\left\langle F^{(\ell)}\left(x^{*}-f^{1}\right), \mathbf{e}_{\iota_{0}}\right\rangle} \\
= & \frac{\left\langle F^{(\ell)}\left(y^{*}-f^{1}\right), \mathbf{e}_{\iota_{0}}\right\rangle}{\left\langle F^{(\ell)}\left(x^{*}-f^{1}\right), \mathbf{e}_{\iota_{0}}\right\rangle}\left(y^{*}-x^{*}\right) .
\end{aligned}
$$

Hence, $x^{*}-f^{1}$ is an eigenvector of the Jacobian, associated with eigenvalue 0 (we discuss this degeneracy at the end of this section), and all $y^{*}-x^{*}$ for fixed points $y^{*} \neq x^{*}$, are eigenvectors as well. Moreover, the associated eigenvalues are the ratios of those of the fixed points themselves, seen as eigenvectors of $F^{(\ell+1)}$. Thus, if all eigenvalues of the latter give fixed points of $\mathcal{M}^{\ell}$, we have all the fixed points of the Jacobian. This is exactly the situation of the binary case, where the eigenanalysis of the Jacobian is known from fixed points of the return map. Thus, we refer to [10] for a discussion about the remaining special cases : eigenvalues of $F^{(\ell+1)}$ that do not correspond to admissible fixed points, and eigenvalues with differing algebraic and geometric multiplicity. Concerning the latter, simple calculations show that when an eigenvalue has geometric multiplicity $>1$, the fixed points given by the associated eigenspace form an affine subspace. This is just as in the binary case (the proof is given in [10], and remains unchanged here ; another result is preserved along with its proof : the fact that straight lines joining fixed points are invariant under $\mathcal{M}^{\ell}$ ).

In any case, the key point is preserved : noting $\mu\left(x^{*}\right)$ the eigenvalue of $F^{(\ell+1)}$ associated with a fixed point $x^{*}$, eigenvalues of the Jacobian have modulus of the 
form $\left|\frac{\mu\left(y^{*}\right)}{\mu\left(x^{*}\right)}\right|$, and thus $x^{*}$ is (asymptotically) stable if $\mu\left(x^{*}\right)$ is a (strict) dominant eigenvalue of $F^{(\ell+1)}$.

We can summarize the above discussion in the following proposition :

Proposition 10. Let $\mathbf{a}=a^{0} \ldots a^{\ell-1} a^{0}$ provide a cyclic sequence of walls in phase space with nonempty returning domain $D_{\mathbf{a}}$, and a return map written in the form (38). Assume the matrix $F^{(\ell+1)}$ has an eigenvector $v$ with real eigenvalue $\mu$. Then, the point :

$$
x^{*}=f^{1}+\frac{\mu}{\left\langle F^{(\ell)} v, \mathbf{e}_{\iota_{0}}\right\rangle} v
$$

is a fixed point of the return map, provided

$$
\frac{\mu}{\Delta}>1, \quad \text { and } \quad x^{*} \in D_{\mathbf{a}} .
$$

Morevoer, it is asymptotically stable if, for any other eigenvalue $\eta$ of $F^{(\ell+1)}$ the following holds :

$$
|\mu|>|\eta| \text {. }
$$

If the inequality is weak, $x^{*}$ is stable, and it is unstable otherwise.

This proposition is an exact analogue of what is known in the case of binary systems, the differences being that eigenvectors are translated by $f^{1}$, and the eigenvalue $\mu$ can be negative. Moreover it must be of greater modulus than $\Delta$, a quantity obtained from the thresholds and focal points coordinates involved in the cycle, instead of being just $>1$ like in the binary case.

Remark 4. This resemblance is intuitively not surprising, and has always been considered true in previous discussions in the literature. Anyway, proving this involves a different way of writing things than usual. In particular, the return map is usually reduced to a $\mathbb{R}^{n-1} \rightarrow \mathbb{R}^{n-1}$ application, since at each step a coordinate is known to be zero. Here, the latter does not hold in general, and the same coordinate $x_{i}$ may take different threshold values along a single orbit. This is why all components of $\mathcal{M}$ have been kept. This also explains why the Jacobian $D \mathcal{M}^{\ell}$ is noninjective : the dynamics is essentially $n-1$ dimensional, and keeping all $n$ components introduces degeneracy. Mathematically, the fact of using a somehow superfluous coordinate has to be related to the use of homogeneous coordinates in the context of projective geometry. In the latter context, the additional coordinate is usually set to 1 , but since here one deals with a piecewise projective mapping, this homogenization must be reiterated in each box, using a corresponding threshold value instead of 1 .

Finally, in the binary case one important result stands, that is lost here. It is the fact that trajectories starting from the same ray through the origin stay on this ray when iterating the mapping $\mathcal{M}$. Hence the long run dynamics can be projected on a $n-1$ sphere without loss of information, which has allowed to prove that no chaotic dynamics may happen in a 3-dimensional binary system [32], as well as to 
study in details a class of 4-dimensional systems [17]. Here there is no apparent way to extend this property, since threshold hyperplanes do not intersect at a single point.

The domains on which iterates of $\mathcal{M}$ are defined, as described in equation (30) and proposition 8 , can be described via paths on an oriented graph. These paths form a symbolic dynamical system, which provides useful tools to investigate the dynamics on $(\mathscr{D}, \mathcal{M})$. It is the aim of the next section to develop this aspect.

\section{The symbolic dynamics approach}

In this section, the uniform decay rates assumption $\mathbf{H 3}$ is not required.

\subsection{The transition graph and its induced codings}

The partition of phase space into boxes naturally induces an oriented graph, with edges representing admissible transitions between boxes. Formally, we denote the transition graph as $\mathrm{TG}=(\mathcal{A}, \mathcal{E})$. It is an oriented graph, whose vertices are subscripts of boxes. Edges correspond to pairs of boxes that are successively crossed by some trajectory, when iterating the transition map $\mathcal{M}$. This includes 1-loops, corresponding to boxes that are forward invariant (i.e. with a subscript in $\mathcal{T}$ ), and pairs that are adjacent through some $n-1$ dimensional threshold hyperplane (i.e. a single wall), ordered in accordance with the flow lines. As we already observed when writing $\mathbf{H} 2, B_{a}$ and $B_{b}$ are adjacent through a single wall if and only if $a-b= \pm \mathbf{e}_{i}$, for some $i \in \mathbb{N}_{n}$. Now, the flow lines in a box $B_{a}$ only escape in directions $i \in I_{\text {out }}(a)$, with monotonicity given by the sign $\pm_{i}=\left(\mathbf{d}_{i}(f(a))-a_{i}\right)$ we have introduced in section 3.2. This sign $\pm_{i}$ depends on the symbol $a$, whose value will be clear from the context in the sequel. In short, $\mathcal{E}$ can thus be written :

$$
\mathcal{E}=\{(a, a) \mid a \in \mathcal{T}\} \cup\left\{\left(a, a \pm_{i} \mathbf{e}_{i}\right) \mid a \in \mathcal{A} \backslash \mathcal{T}, i \in I_{\text {out }}(a)\right\} .
$$

Thus, TG describes transitions between boxes that occur through $n-1$ dimensional faces. Trajectories crossing lower dimensional faces are then ignored in this description, as with the construction of the domain $\mathscr{D}$ on which $\mathcal{M}$ is defined. A nice property of TG is that it is naturally embedded on a cubical lattice, inherited from phase space partition through the double correspondence vertices-boxes and edges-facets. In case of a single threshold per direction, the lattice reduces to a single $n$-cube, whose symmetry properties have been used to improve classification of Glass networks and their dynamics $[9,19]$.

Attractors of the discrete-time, continuous-space system $(\mathscr{D}, \mathcal{M})$ have a counterpart in TG. The converse does not hold in general, and the discrete attractors of TG may be of different nature than those of the original system. Self loops correspond to asymptotically stable steady states, since they arise at boxes containing their own focal point. The cases of attracting cycles and foci - both appearing as loops in TG - have been greatly clarified by several authors [21,22,32,38]. Yet, a complete characterization of those attractors in TG that have an equivalent in phase space is still lacking, and the task remaining in this direction is still a largely 
open problem: although limit cycles can be determined form properties of the map calculated on a loop, very few is known about the possible attractors associated to a given logical structure, in general.

Classically, TG codes a subset of infinite words on the alphabet $\mathcal{A}$, given by infinite paths on this graph. This set is given by :

$$
\mathscr{S}(\mathrm{TG})=\left\{\mathbf{a}=\left(a^{t}\right)_{t \in \mathbb{N}} \mid \forall t \in \mathbb{N},\left(a^{t}, a^{t+1}\right) \in \mathcal{E}\right\} \subset \mathcal{A}^{\mathbb{N}} .
$$

Such an approach is usually referred to as symbolic dynamics, since $\mathcal{A}$ is a finite set whose elements are symbols representing a subset of state space. Moreover, $\mathscr{S}$ can be seen as a metric space on which discrete dynamics can be defined. We shall give the sole ingredients of this theory that will be of direct use here, without detailing its numerous developments. Several textbooks about dynamical systems give an introduction to this subject; here we mainly rely on the classical reference [31]. The dynamics is obtained by introducing the shift operator $\sigma: \mathscr{S} \rightarrow \mathscr{S}$, defined by $(\sigma(\mathbf{a}))^{t}=a^{t+1}$.

This operator is continuous for several metrics, among which the following will be convenient :

$$
\rho(\mathbf{a}, \mathbf{b})= \begin{cases}0 & \text { if } \mathbf{a}=\mathbf{b} \\ 2^{-} \min \left\{t \mid a^{t} \neq b^{t}\right\} & \text { if } \mathbf{a} \neq \mathbf{b}\end{cases}
$$

Thus, the more initial terms of $\mathbf{a}$ and $\mathbf{b}$ coincide, the closer they are for $\rho$. The space $\mathscr{S}$ is compact for $\rho$, and $\sigma$-invariant. As such, it is called a shift space in the literature. The pair $(\mathscr{S}, \sigma)$ constitutes a discrete dynamical system in the usual way. Since orbits of this system are associated with words on the alphabet $\mathcal{A}$, whose elements are in turn representing subsets of the state space of the initial dynamical system, the trajectories of $(\mathscr{S}, \sigma)$ represent sets of trajectories in $(\mathscr{D}, \mathcal{M})$.

The usual way to compare these two dynamical systems requires an application $\phi: \mathscr{D} \rightarrow \mathscr{S}$, which could lead to a conjugation relation of the form : $\phi \circ \mathcal{M}=\sigma \circ \phi$. Depending on $\phi$ being one-to-one or onto it is respectively said to be an embedding or a factor map. If it is bijective with continuous inverse (i.e. a homeomorpism), it is called a (topological) conjugacy. From a topological point of view, two conjugate dynamical systems behave identically, and thus conjugacy is among the strongest equivalence relations one may apply to symbolic dynamical systems. Notably, fixed points, periodic orbits and their period, dense orbits, topological transitivity and topological entropy are well known invariants for conjugacy.

An application like $\phi$ above is clearly linked to the mapping $\mathbf{d}$ introduced in section 2.2, when formalizing H2. d maps points of $\bigcup_{a} \operatorname{int}\left(B_{a}\right)$ onto $\mathcal{A}$. A very similar mapping is introduced in [38], where it serves as a discrete mapping conserving asymptotically stable steady states, and limit cycles for some parameter values when TG essentially consists of a single loop. Another kind of coding has also been used in the case of complicated trajectories in a specific network, with an attractor having the shape of a multiple loop through a given hyperplane [10-12] : a symbol was associated with each loop, and it was shown that a subsequence was not allowed among words on these symbols, for certain parameter values. Such 
dynamics were thus related to the so called golden mean shift (see [31]), and only concerned a subgraph of the whole TG.

Here, $\mathscr{D}$ entirely lies in the complement of d's domain. But it is also contained in the reunion of all facets of boxes $B_{a}$, taken without their boundary. Any of these open facets is well defined by the two boxes it is part of. On the boundary $\partial \mathcal{U}$ of the whole domain this would not work as such, but this boundary can not be reached from the rest of $\mathscr{D}$, so that we can ignore it without much trouble : by $\mathscr{D}$ we now mean $\mathscr{D} \backslash \partial \mathcal{U}$. Then, for all $x \in \mathscr{D}$, either there is a unique pair $(a, b)$ such that $x \in \partial B_{a}^{\text {out }} \cap \partial B_{b}^{\text {in }}$, or some $a \in \mathcal{T}$ such that $x \in \partial B_{a} \cup\{f(a)\}$. Accordingly, we can define a mapping $\Phi: \mathscr{D} \rightarrow \mathcal{E}$, with

$$
\Phi(x)=\left\{\begin{array}{l}
(a, b) \text { if } x \in \partial B_{a}^{\text {out }} \cap \partial B_{b}^{\text {in }} \\
(a, a) \text { if } x \in \partial B_{a} \cup\{f(a)\}, \text { for } a \in \mathcal{T} .
\end{array}\right.
$$

Thus $\Phi$ codes with labels of the edges of TG, instead of vertices. Observe that $\Phi^{-1}(a, b)$ is a practical way to denote the open wall between two adjacent boxes $B_{a}$ and $B_{b}$, respecting the orientation of the flow.

This leads to consider a new shift space, which is obtained from $\mathscr{S}$ through the so called 2-block map $\beta_{2}$, defined by

$$
\left(\beta_{2}(\mathbf{a})\right)^{t}=\left[\begin{array}{l}
a^{t} \\
a^{t+1}
\end{array}\right] \in \mathcal{E} .
$$

This vertical writing of edges will make things clearer when dealing with a full sequence of pairs. We note $\mathscr{S}^{[2]} \doteq \beta_{2}(\mathscr{S}) \subset \mathcal{E}^{\mathbb{N}}$; it is a shift space. The shift operator on $\mathscr{S}^{[2]}$ is noted $\sigma_{[2]}$. Then, the two symbolic dynamical systems $(\mathscr{S}, \sigma)$ and $\left(\mathscr{S}^{[2]}, \sigma_{[2]}\right)$ are conjugate, i.e. $\beta_{2}$ is continuous and $\beta_{2} \circ \sigma=\sigma_{[2]} \circ \beta_{2}$ (cf. [31] p.18). Thus, both systems yield the same information, at least topologically. The latter is more directly related to $\mathcal{M}$ 's dynamics, which is from walls to walls, rather than boxes to boxes, while the first one is more closely related to the dynamics induced by TG since the vertices of this graph are labeled (with symbols from $\mathcal{A}$ ), not its edges.

Now to code the trajectories of $(\mathscr{D}, \mathcal{M})$, we proceed in two steps. First, following [15], one introduces the mapping $\xi: \mathscr{D} \rightarrow \mathscr{D}^{\mathbb{N}}$, defined by :

$$
\xi(x)=\left(x, \mathcal{M} x, \mathcal{M}^{2} x, \ldots\right) .
$$

As mentioned in section 3.3, $\mathcal{M}$ is continuous on $\mathscr{D}$. It is proved in [15] that the mapping $\xi$ above is then a conjugacy, when restricting the range to $\xi(\mathscr{D})$. This proof is done using the following metric on $\mathscr{D}^{\mathbb{N}}$ :

$$
\varrho(\mathbf{x}, \mathbf{y})=\sum_{k \in \mathbb{N}} \frac{1}{2^{k}} \frac{\delta\left(x^{k}, y^{k}\right)}{1+\delta\left(x^{k}, y^{k}\right)},
$$

where $\delta(\cdot, \cdot)$ is any metric on $\mathscr{D}$. The shift operator on $\mathscr{D}^{\mathbb{N}}$ is noted $\sigma_{\mathscr{D}}$.

The second step is a mapping $\Phi_{\infty}: \mathscr{D}^{\mathbb{N}} \rightarrow \mathscr{S}^{[2]}$, which is naturally induced by $\Phi$ :

$$
\Phi_{\infty}\left(\left(x^{k}\right)_{k \in \mathbb{N}}\right)=\left(\Phi\left(x^{k}\right)\right)_{k \in \mathbb{N}}
$$


This application maps sequences on $\mathscr{D}$ to sequences on $\mathcal{E}$, which can be seen as coding infinite words on an uncountable alphabet, with infinite words on a finite alphabet. It is thus the step at which an approximation is done in the process of coding the dynamics.

The two previous steps provide us with a mapping

$$
\phi=\Phi_{\infty} \circ \xi: \mathscr{D} \rightarrow \mathscr{S}^{[2]} .
$$

This mapping can be described in more detail. For this one extends the definition of eq. (30) for domains $D_{\mathbf{a}}$ when a is an infinite word in $\mathscr{S}$ :

$$
D_{\mathbf{a}}=\bigcap_{i \in \mathbb{N}} \mathcal{M}^{-i}\left(\Phi^{-1}\left(a^{i}, a^{i+1}\right)\right) .
$$

The resulting domains are then defined by an infinite set of inequalities like those given in proposition 8 . One has moreover the following result

Proposition 11. The mapping $\phi$ takes constant values on the domains $D_{\mathbf{a}}$ defined in eq. (43). The latter are exactly the connected components of $\mathscr{D}$, hence $\phi$ is continuous.

Proof. Given $\epsilon=\left(\epsilon^{i}\right)_{i \in \mathbb{N}} \in \mathscr{S}^{[2]}$, the preimage $\Phi_{\infty}^{-1}(\epsilon)$ is the product of walls : $\prod_{i \in \mathbb{N}} \Phi^{-1}\left(\epsilon^{i}\right)$.

This product is a subset of the range of $\xi$. Then, noting $\mathbf{a}=\beta_{2}^{-1}(\boldsymbol{\epsilon})$, it follows from the definitions that the $\xi$ preimage of the product above is exactly $D_{\mathbf{a}}$.

Thus, one gets $\phi^{-1}(\epsilon)=\xi^{-1} \circ \Phi_{\infty}^{-1}(\epsilon)=D_{\mathbf{a}}$, and the latter are the preimages of infinite words in $\mathscr{S}^{[2]}: \phi$ is constant on such domains.

Now, each set $\Phi^{-1}\left(a^{0}, a^{1}\right)$, for $\left(a^{0}, a^{1}\right) \in \mathcal{E}$, is either in the relative interior of a wall, or it is a focal point inside its own box. In either case, it is disjoint from all other sets of the same form. Thus, connected components of $\mathscr{D}$ must be subsets of the preimages of $\Phi$. For an infinite word $\mathbf{a}=\left(a^{0}, a^{1} \ldots\right)$, one has $D_{\mathbf{a}} \subset \Phi^{-1}\left(a^{0}, a^{1}\right)$. Then, from the definition of $\mathscr{D}$, eq.(28): $\mathscr{D}=\bigcup_{a \in \mathcal{A}} \operatorname{Dom}\left(\mathcal{M}^{a}\right) \backslash \bigcup_{i \in \mathbb{N}} \mathcal{M}^{-i}\left(\mathscr{F}_{2}\right)$, it is clear that the only obstacles to connectedness of domains $D_{\mathbf{a}}$ are the sets $\mathcal{M}^{-i}\left(\mathscr{F}_{2}\right)$. But faces in $\mathscr{F}_{2}$ are the intersections of two walls or more. Hence in any set intersecting $\mathcal{M}^{-i}\left(\mathscr{F}_{2}\right)$ for some $i$, there are points whose $i$ th iterates lie in distinct walls. It follows that the connected components are exactly the sets of points following the same itinerary, i.e. the preimages of $\phi$, which we just proved to be the $D_{\mathbf{a}}$ s.

All constructions above may be summarized using the following diagram, in which all paths commute :

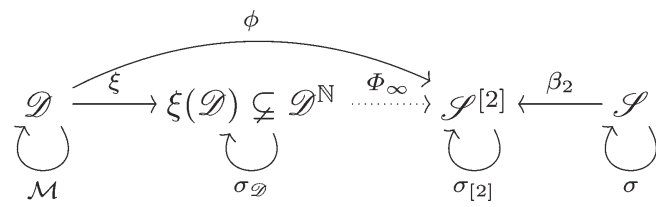



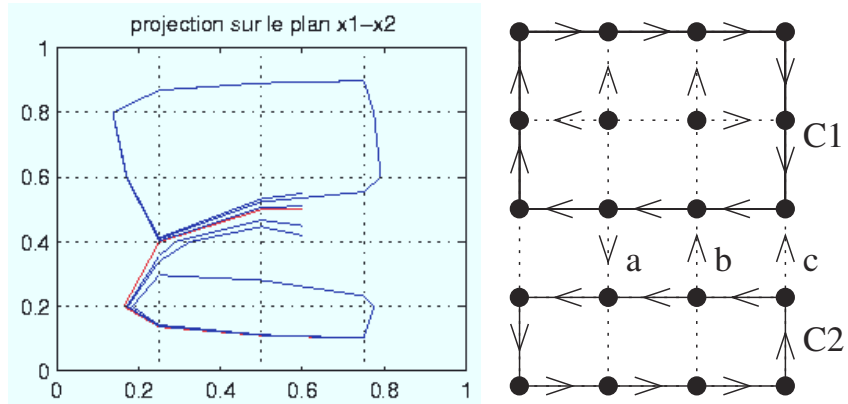

Fig. 7. Example of a system in $\mathbb{R}^{2}$ with two limit cycles. The phase plane is on the left, and the transition graph on the right. On this latter, $C 1$ and $C 2$ are obvious abbreviations for cyclic sequences of walls. a and $b$ are also shorthands for symbols in $\mathcal{A}^{2}=\left(\mathbb{N}_{4} \times \mathbb{N}_{5}\right)^{2}$. As an illustration of the violation of injectivity of the operator $\phi$ it appears here that both coexisiting cycles are attracting for a whole set of orbits crossing similar sequences of walls. Surjectivity is lacking as well, due to the fact that all words in the language $\left(C 1^{*} \mathrm{a} C 2^{*} \mathrm{~b}\right)^{*}$ are allowed blocks in $\mathscr{S}^{[2]}(\mathrm{TG}$ ), while there is no continuous trajectory looping around both cycles $C 1$ and $C 2$

Now, the problem is that the left part of the diagram is clearly not a conjugacy, a fact we have stressed using dotted arrows at the noninvertible step. Actually $\Phi_{\infty}$, and thus $\phi$, are neither one-to-one nor onto in general, as illustrated for instance on figure 7 and its legend.

Remark 5. One can see on the transition graph above, that some edges are not oriented. They correspond to white walls in the phase plane, which are unavoidable inside cycles of the plane. White walls are less problematic than black walls, since they are not reachable in increasing time; they are unstable surfaces in phase space. This suggests that our $\mathbf{H} \mathbf{2}$ is too strong, and precludes interesting dynamics. A weaker version of this condition should only forbid black walls. However, most interesting dynamics appear in higher dimensional spaces, where white walls do not necessarily occur inside periodic trajectories.

The non-injectivity of $\phi$ is an inevitable feature of the system $(\mathscr{D}, \mathcal{M})$, in which the domains $D_{\mathbf{a}}$ associated with admissible itineraries are not reduced to single points. The fact that $\phi$ is not surjective, on the other hand, means that some infinite paths in the transition graph do not correspond to any admissible trajectory of the continuous system. According to this, the (generally proper) subset $\phi(\mathscr{D}) \subset \mathscr{S}^{[2]}$ is exactly the space of admissible trajectories in TG, thus it seems worthwhile to study the subsystem $\left(\phi(\mathscr{D}), \sigma_{[2]}\right)$. First, one must of course check that this system is well-defined. From the commuting diagram (44), $\phi(\mathscr{D})$ inherits shift-invariance from $\mathscr{D}$ 's $\mathcal{M}$-invariance. Now, the space $\phi(\mathscr{D})$ must be compact in order to get a properly defined symbolic dynamical system. Since $\mathscr{S}^{[2]}$ is compact and contains $\phi(\mathscr{D})$, we only have to check whether the latter is closed.

It happens that $\phi(\mathscr{D})$ is not closed in general. Actually, there are two equivalent characterizations of shift spaces : they can be defined as shift-invariant compact subspaces of the full shift, or as subspaces of all infinite words on the alphabet 
defined by a (possibly infinite) set of forbidden (finite) blocks. The equivalence of these two characterizations is proven in [31], pp. 5-6 and 179. Here, proposition 11 implies that $\phi(\mathscr{D})$ is the set of words $\epsilon=\beta_{2}(\mathbf{a})$ such that $D_{\mathbf{a}} \neq \varnothing$. Hence, forbidden words in $\phi(\mathscr{D})$ are exactly those for which $D_{\mathbf{a}}=\varnothing$. Letting $D_{\mathbf{a}}^{i}$ be the finite intersection $D_{\mathbf{a}}^{i}=\bigcap_{j=0}^{i} \mathcal{M}^{-j}\left(\Phi^{-1}\left(a^{j}, a^{j+1}\right)\right)$, one sees that $D_{\mathbf{a}}=\bigcap_{i \in \mathbb{N}} D_{\mathbf{a}}^{i}$ is empty if either one $D_{\mathrm{a}}^{i}$ is empty as well, or else all of them are nonempty, but their full intersection is. In the latter case $\beta_{2}$ (a) is an infinite word that is forbidden in $\phi(\mathscr{D})$, while all its subwords are allowed. Thus, it does not satisfy the first characterization of a shift space.

We are then naturally led to consider the system $\left(\overline{\phi(\mathscr{D})}, \sigma_{[2]}\right)$. This one is a properly defined symbolic dynamical system, since the closure $\overline{\phi(\mathscr{D})}$ is clearly compact. In fact, it is not difficult to prove that :

$$
\overline{\phi(\mathscr{D})}=\beta_{2}\left(\left\{\mathbf{a} \in \mathscr{S} \mid \forall i \in \mathbb{N}, D_{\mathbf{a}}^{i} \neq \varnothing\right\}\right) .
$$

As a justification, note that the set above is the one that is defined as a coding space for a general dynamical system in [31], p. 202. Furthermore, a similar coding is used for a class of dynamical systems having the same kind of discontinuities as the one we consider : piecewise isometries. For these systems, topological entropy is defined to be the entropy (see next section) of the exact analogue of what we call here $\left(\overline{\phi(\mathscr{D})}, \sigma_{[2]}\right)$, see [4].

Now, the dynamics of the two symbolic dynamical systems $\left(\overline{\phi(\mathscr{D})}, \sigma_{[2]}\right)$ and $\left(\mathscr{S}^{[2]}, \sigma_{[2]}\right)$ may be compared, which is done in the next section in terms of topological entropy.

\subsection{Comparison of topological entropies}

Topological entropy is an important tool in symbolic dynamics. Actually, this quantity is conjugacy invariant, and can be effectively computed for systems described by an oriented graph. We first recall a few definitions and properties. Proofs and additional details may be found in chapters 4 and 6 of [31], along with an extensive bibliography. We will often omit the adjective topological, and simply use the term entropy in this section.

Let $X$ be a shift space, $\sigma_{X}$ the shift operator on $X$, and $\mathcal{L}_{k}(X)$ the set of blocks of length $k$ appearing in $X$. Then, the entropy of $\left(X, \sigma_{X}\right)$ is defined as :

$$
h\left(X, \sigma_{X}\right)=\lim _{k \rightarrow \infty} \frac{1}{k} \log \left(\# \mathcal{L}_{k}(X)\right),
$$

where $\log$ is conventionally the logarithm with base 2 . This quantity is nonnegative, and its positivity is a common criterion for the existence of chaos in a dynamical system.

In case when $X$ is defined by the way of infinite paths on an oriented graph $G$, let $A$ be the adjacency matrix of $G: A_{i j} \in\{0,1\}$, and $A_{i j}=1$ iff $(i, j)$ is an edge in the graph. Define the irreducible components of $A$ as the equivalence classes for the equivalence relation : $i \sim j$ if $\exists p, q \in \mathbb{N},\left(A^{p}\right)_{i j} \neq 0$ and $\left(A^{q}\right)_{j i} \neq 0$. This corresponds exactly to strongly connected components in $G$. Let $A_{i}, i=1 \ldots k$ 
be the submatrices of $A$ with all indices in the same equivalence class. If there is a single class, A is said to be irreducible.

The Perron-Frobenius theorem ensures that any matrix with nonnegative entries has a dominant positive eigenvalue $\mu_{A}$, which is simple, and is associated with a nonnegative eigenvector. Following [31], we call the Perron eigenvalue of $A$ the maximum : $\mu_{A} \doteq \max _{i=1 \ldots k} \mu_{A_{i}}$.

Then, the entropy is given by :

$$
h\left(X, \sigma_{X}\right)=\log \mu_{A} .
$$

We will need the following lemma, proved in [31] as theorem 4.4.7, p.123.

Lemma 3. Let $A$ be an irreducible matrix, and $0 \leqslant B \leqslant A$, with $B_{i j}<A_{i j}$ for $a$ pair $i, j$ of indices. Then $\mu_{B}<\mu_{A}$.

Since $\mathscr{S}$ and $\mathscr{S}^{[2]}$ are conjugate they have the same entropy. As $\mathscr{S}$ is exactly the shift space induced by infinite paths on TG, one simply notes $h_{\mathrm{TG}}=h\left(\mathscr{S}^{[2]}, \sigma_{[2]}\right)=$ $h(\mathscr{S}, \sigma)$. One also abbreviates : $h_{\phi(\mathscr{D})}=h\left(\overline{\phi(\mathscr{D})}, \sigma_{[2]}\right)$. Now, from the fact that $\overline{\phi(\mathscr{D})} \subset \mathscr{S}^{[2]}$, one can only infer $h_{\phi(\mathscr{D})} \leqslant h_{\mathrm{TG}}$. The next theorem yields a stronger result when the transition graph contains a splitting box that is 'inside' the domain, with certain conditions.

We use $[a]$ to denote the $\sim$ equivalence class of $a$, i.e. the set of vertices in the same strongly connected component of TG as $a$. Observe that such components are either single vertices, loops, or more complex structure involving several intersecting loops. Note also that the adjacency matrix of TG is indexed by $\mathcal{A}$, and not by integers.

Theorem 1. Let TG be the transition graph associated with the dynamical system $(\mathscr{D}, \mathcal{M})$ obtained from a piecewise affine system of the form (1), and satisfying $\mathbf{H 1}$ and $\mathbf{H} 2$.

Suppose moreover that there is an $a \in \mathcal{A}$, and at least two distinct directions $i_{1}, i_{2} \in I_{\text {out }}(a)$, such that for $j \in\{1,2\}$ and $\pm \in\{-,+\}$,

$$
a \pm \mathbf{e}_{i_{j}} \in[a] .
$$

Finally, assume that besides [a], all equivalence classes are either loops, single vertices, or contain a vertex satisfying the same conditions as a.

Then,

$$
h_{\phi(\mathscr{D})}<h_{\mathrm{TG}} .
$$

Proof. To simplify the discussion, we assume without loss of generality that $\pm_{i_{j}}=$ + , for $j \in\{1,2\}$.

Since $i_{1}$ and $i_{2}$ are exiting directions for $a$, and since $\mathbf{H} \mathbf{2}$ precludes white walls, the pairs $\left(a-\mathbf{e}_{i_{j}}, a\right)$ and $\left(a, a+\mathbf{e}_{i_{j}}\right)$, for both values of $j$, are edges in TG. All vertices appearing in these four edges being in the same strongly connected component, there must be a path from $a+\mathbf{e}_{i_{j}}$ to $a-\mathbf{e}_{i_{j}}$, for both $j$. Hence, there are necessarily two loops of the form

$$
a+\mathbf{e}_{i_{j}} \rightarrow \cdots \rightarrow a-\mathbf{e}_{i_{j}} \rightarrow a \rightarrow a+\mathbf{e}_{i_{j}},
$$

which intersect at vertex $a$. 
Note that loops (including single vertices, seen as 1-loops) have zero entropy, since they only generate periodic words. From its definition, entropy is always nonnegative. Then from eq. (46), the entropy of TG must be $\log \mu_{A_{[a]}}$, for the $a$ above, or one fulfilling the same requirements. From now on, let $[a]$ be the class with maximal Perron eigenvalue : $\mu_{A}=\mu_{A_{[a]}}$.

Now, lemma 1 ensures that at most one of the two domains $D_{i_{1} i_{1}}, D_{i_{2} i_{2}}$ is nonempty, where we define these domains as in eq. (14), at the box $B_{a}$. This means exactly that one of the two 2-blocks :

$$
\left[\begin{array}{c}
a-\mathbf{e}_{i_{j}} \\
a
\end{array}\right]\left[\begin{array}{c}
a \\
a+\mathbf{e}_{i_{j}}
\end{array}\right], \quad j \in\{1,2\},
$$

is a forbidden block in the shift space $\overline{\phi(\mathscr{D})}$. Suppose for example that $D_{i_{1} i_{1}}=\varnothing$. Since this restricts the allowed 2-blocks, we turn our attention to $\mathscr{S}^{[2]}$ instead of $\mathscr{S}$. As both shift spaces are conjugate, they have common entropy. Moreover, $\mathscr{S}^{[2]}$ can also be described by an oriented graph, whose vertices are given by edges in $\mathrm{TG}$, and edges are given by those pairs of edges $(e, f)$ in TG such that the terminal vertex of $e$ is the initial vertex of $f$.

In this new graph, denoted $\mathrm{TG}^{[2]}$, a quick inspection shows that strongly connected components that are loops or single vertices correspond to connected components of the same nature in TG, although their number may differ. Similarly, other strongly components of TG correspond to strongly connected components in $\mathrm{TG}^{[2]}$ that are not loops. Hence, the class in $\mathrm{TG}^{[2]}$ which corresponds to $[a]$ is the class $\left[\left(a-\mathbf{e}_{i_{j}}, a\right)\right]=\left[\left(a, a+\mathbf{e}_{i_{j}}\right)\right]$, for both values of $j$.

Note $A^{[2]}$ the adjacency matrix of $\mathrm{TG}^{[2]}$. Its subscripts are thus pairs of edges. It admits an irreducible submatrix with indices in the class $\left[\left(a-\mathbf{e}_{i_{j}}, a\right)\right]$. We denote it $A_{[a]}^{[2]}$. In this submatrix, the entries $\left(a-\mathbf{e}_{i_{j}}, a\right),\left(a, a+\mathbf{e}_{i_{j}}\right)$ are equal to 1 for both $j$. Set the entry with indices $\left(a-\mathbf{e}_{i_{1}}, a\right),\left(a, a+\mathbf{e}_{i_{1}}\right)$ to zero. One gets a matrix $B_{[a]}^{[2]}$, such that $\mu_{B_{[a]}^{[2]}}<\mu_{A_{[a]}^{[2]}}$ by virtue of lemma 3 .

Since we have supposed $D_{i_{1} i_{1}}=\varnothing, \overline{\phi(\mathscr{D})}$ is a subset of the shift space induced by matrix $B^{[2]}$, obtained from $A^{[2]}$ after setting elements to zero as described above, for all classes $[a]$ that are not loops nor single vertices. Hence,

$$
h_{\phi(\mathscr{D})} \leqslant \log \mu_{B^{[2]}} .
$$

The Perron eigenvalue of $A^{[2]}$ is the same than that of $A$, and it is clearly that of the irreducible component $A_{[a]}^{[2]}$. On the other hand, the Perron eigenvalue of $B^{[2]}$ is given by one of its irreducible components $B_{[b]}^{[2]}$, where $[b]$ may differ from $[a]$. In any case, one gets a sequence of inequalities :

$$
\mu_{B^{[2]}}=\mu_{B_{[b]}^{[2]}}<\mu_{A_{[b]}^{[2]}} \leqslant \mu_{A_{[a]}^{[2]}}=\mu_{A^{[2]}}=\mu_{A} .
$$

Combining these inequalities with (47), one gets :

$$
h_{\phi(\mathscr{D})}<\log \mu_{A}=h_{\mathrm{TG}} \text {. }
$$


In words, theorem 1 means that the dynamics on the transition graph is much more complicated than the dynamics allowed in the continuous dynamical system $(\mathscr{D}, \mathcal{M})$, as discussed in the conclusion. It requires special conditions on $\mathrm{TG}$, namely the existence of a particular kind of splitting box in the 'most complicated' region of phase space. But it is a very general result in the sense that it holds for any parameter values yielding the same transition graph. Hence, it provides a strict upper bound for the complexity of a continuous system, which can be read directly from the discrete structure of TG, without detailed knowledge of parameter values. Furthermore, in the case when TG is strongly connected, local information on a vertex $a \in \mathcal{A}$ provides a result on entropy, which is a global characteristic of the system. Yet, another limitation of this result is that the required kind of splitting box can only happen in a system with at least two thresholds in at least two directions. This is counterbalanced by the fact that many biological systems are known to involve several thresholds per variable.

\subsection{Example in $\mathbb{R}^{3}$}

We now examine a three dimensional example that will serve as an illustration of results in this paper. Namely, we consider a transition graph TG which contains three cycles $\mathcal{C}^{1}, \mathcal{C}^{2}, \mathcal{C}^{3}$, sharing a single vertex $a$, and only loops or single vertices as other strongly connected components. It follows that $B_{a}$ must have three escaping directions : $I_{\text {out }}(a)=\mathbb{N}_{3}$, like in figures 5 and 6 . In the following, we will abusively identify cycles in TG and their corresponding sequence of boxes in phase space. To simplify formulation, let $\pm_{i}=+$ for $i \in \mathbb{N}_{3}$. We denote

$$
\mathcal{C}^{i}=a \rightarrow a^{i 1} \rightarrow \cdots \rightarrow a^{i \ell-1} \rightarrow a,
$$

where $a^{i 1}=a+\mathbf{e}_{i}$.

In accordance with lemma 1 , we put $D_{11} \neq \varnothing$, and $D_{22}=D_{33}=\varnothing$. In order to focus on this triple loop structure, we assume moreover that $B_{a}$ is the only box that is splitting among those crossed by the three circuits. Thus, no trajectory can escape $\bigcup_{i} \mathcal{C}^{i}$ : it is an invariant subset in phase space. As a last assumption, all cycles have the same length $\ell$, so that $\mathcal{M}^{\ell}: \partial B_{a}^{i n} \rightarrow \partial B_{a}^{i n}$ is a properly defined return map.

With these sole assumptions, one can readily illustrate theorem 1 . First, a known fact about entropy is that $h\left(X, \sigma^{\ell}\right)=\ell h(X, \sigma)$, for any symbolic dynamical system $(X, \sigma)$. Hence, the inequality provided in the theorem holds iff the same holds for $\ell$-steps dynamics. For the latter, a transition graph may be formed : $\mathrm{TG}^{\ell}$ has adjacency matrix $A^{\ell}$ where $A$ is that of TG. The subset of $\mathrm{TG}^{\ell}$ formed by the cycles is not strongly connected as $\bigcup_{i} \mathcal{C}^{i}$. Yet, since $\sigma^{\ell}(a)=a$, and for any pair $i, j,\left(\sigma^{\ell}\right)^{-1}\left(a^{i j}\right)=\left\{a^{1 j}, a^{2 j}, a^{3 j}\right\}$, strongly connected components take the form presented in Fig. 8.

Associating a label $i$ for cycle $\mathcal{C}^{i}$, one is led to a labelling of the edges of the graph on the left in figure 8, and a labelling of the vertices of the graph on the right. In both cases, the induced shift space is the full 3-shift $\left(\mathbb{N}_{3}\right)^{\mathbb{N}}$. In the following, we use the vertex labeled graph on the right, for it is more convenient. The entropy of the full 3-shift is readily computed, yielding $\log 3$. Thus $h_{\mathrm{TG}}=\frac{1}{\ell} \log 3$. 

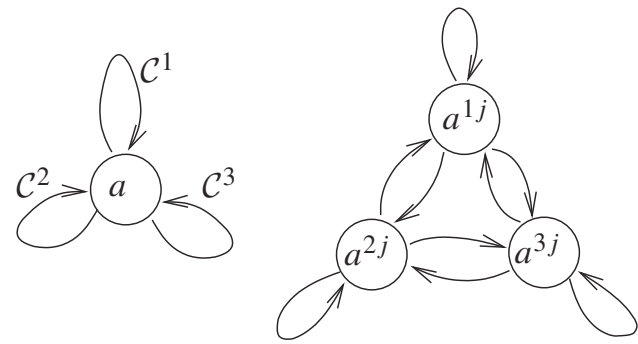

Fig. 8. Subgraph of $\mathrm{TG}^{\ell}$ corresponding to $\bigcup_{i} \mathcal{C}^{i}$. Vertex $a$ is fixed, but it can attain itself through any of the three cycles, as illustrated by the use of three self loops. For any $j \in \mathbb{N}_{\ell-1}$, the three vertices $a^{1 j}, a^{2 j}, a^{3 j}$ form a graph like that on the right above

Now, since

$$
D_{22}=D_{33}=\varnothing \text {, }
$$

the vertex $a^{2 j}$ (resp. $a^{3 j}$ ) can not be reached via cycles having $a-\mathbf{e}_{2}\left(\right.$ resp. $\left.a-\mathbf{e}_{3}\right)$ as last vertex. Actually, recall that the first vertex of each cycle $\mathcal{C}^{i}$ is $a+\mathbf{e}_{i}$. From this and the fact that the three cycles do not share any vertex except $a$, it follows that there exist some permutation $\pi: \mathbb{N}_{3} \rightarrow \mathbb{N}_{3}$, such that for all $i, a^{i \ell-1}=a-\mathbf{e}_{\pi(i)}$. Then, (48) implies that both edges $\left(a^{\pi^{-1}(2) j}, a^{2 j}\right)$ and $\left(a^{\pi^{-1}(3) j}, a^{3 j}\right)$ are irrelevant with respect to the dynamics in $\overline{\phi(\mathscr{D})}$.

In other words, instead of an adjacency matrix with all entries set to 1 , the shift space $\overline{\phi(\mathscr{D})}$ is a subset of the shift induced by an oriented graph whose adjacency matrix have zero entries at $\pi^{-1}(2), 2$ and $\pi^{-1}(3), 3$. The permutation $\pi$, may have 6 different values, but symmetries lead to only three qualitatively distinct cases. These cases respectively lead to the following dominant eigenvalue (computed using Maple9.5) :

$$
\begin{aligned}
& 1+\sqrt{2} \approx 2.41421 \\
& 1+\frac{\sqrt[3]{108+12 \sqrt{69}}+\sqrt[3]{108-12 \sqrt{69}}}{6} \approx 2.32472
\end{aligned}
$$

et

$$
1+\frac{\sqrt[3]{28+84 \sqrt{3} i}+\sqrt[3]{28-84 \sqrt{3} i}}{6} \approx 2.24698
$$

In all cases, the value is lower than 3 , illustrating theorem 1.

Observe that no explicit values of parameters in $(\mathscr{D}, \mathcal{M})$ have been fixed. The only requirements concern the boxes in which focal points lie. We also have supposed $D_{11} \neq \varnothing$, but lemma 1 guarantees that there is at most one $i$ with nonempty $D_{i i}$, which would have led us to the same conclusion for any value of $i$.

Now, it would be interesting to study the continuous dynamics in more detail. Notably, the upper bound $\frac{1}{\ell} \log (\mu)$ is positive for the three possible values of $\mu$, which is characteristic of chaotic systems. With this in mind, numerical simulation was carried out on an example, with the transition graph of figure 9. This 


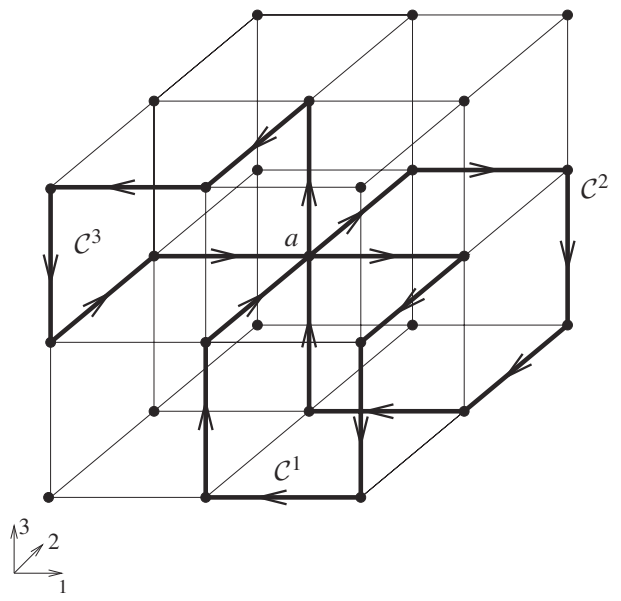

Fig. 9. The transition graph chosen for numerical investigations. Edges with no arrow point towards the cycles. Remark that some of them are not well oriented : they correspond to white walls in phase space. This is not necessary in $\mathbb{R}^{3}$, provided there are enough thresholds. As we deal here with an illustrative example, we have chosen to use no more than 2 threshold per variable. Then, constructing 3 cycles of length $\ell=6$ required white walls, which have anyway no influence on the dynamics inside $\bigcup_{i} \mathcal{C}^{i}$

corresponds to the permutation

$$
\pi=\left(\begin{array}{lll}
1 & 2 & 3 \\
2 & 3 & 1
\end{array}\right),
$$

and thus to the adjacency matrix

$$
\left[\begin{array}{lll}
1 & 0 & 1 \\
1 & 1 & 0 \\
1 & 1 & 1
\end{array}\right],
$$

which has the second value above as dominant eigenvalue, i.e. approximately 2.32472 .

All focal points were fixed with values in agreement with TG, except $f(a)=$ $f(222)$, the focal point of the splitting box. We simulated about one hundred systems, each with a fixed random value of $f(a)$, constrained in the box $B_{333}$. For each value of the focal point, 10 initial conditions where chosen randomly in $B_{222}$, and a 200-step simulation carried.

This only led us to phase portraits composed of one to three limit cycles, with a majority of portraits with three cycles. Presumably, such phase portraits correspond to a zero topological entropy. A typical example of such portraits is shown in figure 10. Anyway, it is commonly known that chaotic trajectories are not easily captured by numerical simulation. Moreover, all evidence of chaotic behaviour in systems like those we study here appeared in dimension 4 or more. With a single threshold per direction, we already mentioned that chaos is not possible in $\mathbb{R}^{3}$. We 

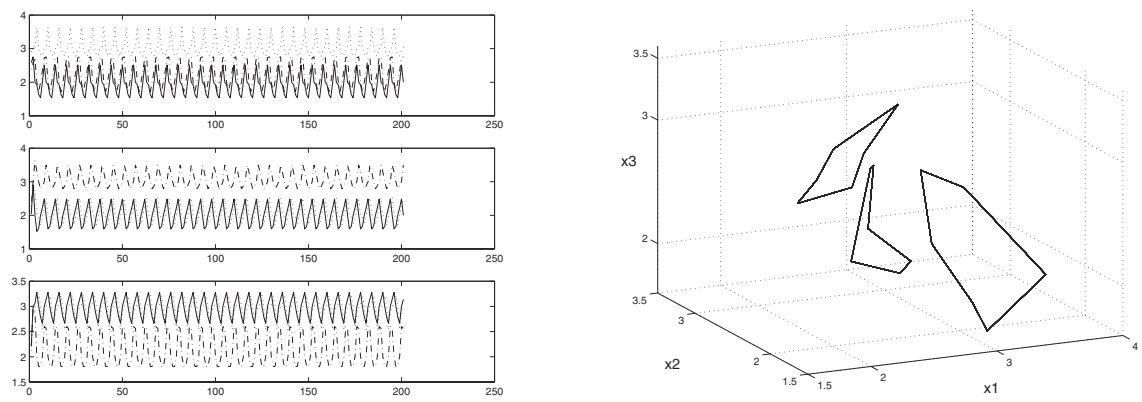

Fig. 10. Three limit cycles of the same system. On the left, the superposed coordinates of a vector versus time, for three different initial conditions. On the right, the corresponding orbits in $\mathbb{R}^{3}$, without transients for improved visualization. Thresholds are $\{2,3\}$ in all directions

plan to analyse further some typical examples like that of figure 9, with the aid of tools and results of this paper, as well as more systematic numerical simulations.

\section{Conclusion}

Besides extending formerly known results to a more general context, the present work provides a geometric framework to study systems of the form (1). The main result obtained with the help of this framework is theorem 1, which states that the transition graph alone is much too coarse to properly describe dynamics of the continuous system. Actually, since entropy measures the growth rate of distinguishable orbits, the inequality provided in theorem 1 means that, asymptotically, the diversity of orbits in a piecewise affine system is neglictible compared to its discrete analogue. The choice of topological entropy as a criterion arises because this quantity can be computed for dynamical systems induced by an oriented graph. On the other hand, the case when TG is really too approximate, i.e. when theorem 1 applies, can be read directly from the graph, without knowing precise parameter values. Hence, it leads us to consider TG as a good source of information, provided it is closely examined.

As all frameworks, it opens several directions for future research. Among them, the study of examples like that of section 5.3 must be pursued. Proposition 10 on the return maps, as well as the explicit description of returning domains $D_{\text {a }}$ provided in proposition 8 , shall be useful results for numerical investigation. They have equivalent statements in the binary case, that have already proved their ability to improve our knowledge of Glass systems.

Another direction concerns topological entropy, and is twofold. First, some additional information for specific systems may improve theorem 1, which makes few assumptions on parameter values. More constraining requirements should help to determine more forbidden blocks in $\overline{\phi(\mathscr{D})}$. Here again, proposition 8 shall help, since we have seen that forbidden blocks are exactly those finite words a for which $D_{\mathbf{a}}$ is empty. An other work remaining would be a more detailed comparison of the dynamics in $(\mathscr{D}, \mathcal{M})$ and $\left(\overline{\phi(\mathscr{D})}, \sigma_{[2]}\right)$. A first difficulty, is that $\mathscr{D}$ is not compact, 
and the map $\mathcal{M}$ can not be extended to the closure $\overline{\mathscr{D}}=\bigcup_{a} \operatorname{Dom}\left(\mathcal{M}^{a}\right)$. Although definitions exist for the topological entropy of noncompact sets $[3,25,35]$ they are less easily computed than that of an oriented graph. A better way to handle this question would be to consider trajectories that intersect lower dimensional faces. Then, the domain of the dynamics would be the compact set $\overline{\mathscr{D}}$. Moreover, we have met twice the presence of white walls inside cycles, which could indicate that excluding such walls is too strong a hypothesis. There are mainly two approaches to the problem of dynamics in codimension 2 faces. One consists in comparing the piecewise affine dynamics with the smooth one it is inspired by. This is done with tools from singular perturbation theory, the most complete and recent reference on these techniques being [37]. The other one uses the Filippov notion of solution for a differential equation with discontinuous right-hand side [6,23]. The principle is to replace the differential equations by differential inclusions at lower dimensional faces. This latter approach seems more closely related to the qualitative flavour of symbolic dynamics than singular perturbation techniques. Moreover, the mapping $\Phi_{\infty}$ we have used in the process of coding the dynamics sends full sets of trajectories to infinite words. Hence, extending it to solutions of differential inclusions does not seem out of reach, at least in principle.

Acknowledgements. This research was partially supported by the Région Rhône-Alpes, CALCEL project. This work was performed while the author was preparing a PhD thesis in the LMC-IMAG laboratory, Grenoble, France, under the supervision of Pr. J. Della Dora. The author would like to thank one of the anonymous referees for his helpful and very detailed comments.

\section{References}

1. Albert, R., Barabási, A.L.: Statistical mechanics of complex networks. Reviews of Modern Phys. 74, 47 (2002)

2. Bagley, R.J., Glass, L.: Counting and classifying attractors in high dimensional dynamical systems. J. Theor. Biol. 183, 269-284 (1996)

3. Bowen, R.: Topological entropy for noncompact sets. Trans. Am. Math. Soc. 184, 125136 (1973)

4. Buzzi, J.: Piecewise isometries have zero topological entropy. Ergod. Theor. Dyn. Sys. 21, 1371-1377 (2001)

5. Cinquin, 0., Demongeot, J.: Positive and negative feedback: striking a balance between necessary antagonists. J. Theor. Biol. 216 (2), 229-241 (2002)

6. de Jong, H., Gouzé, J.-L., Hernandez, C., Page, M., Sari, T., Geiselmann, J.: Qualitative simulation of genetic regulatory networks using piecewise-linear models. Bull. Math. Biol. 66 (2), 301-340 (2004)

7. de Jong, H., Gouzé, J.-L., Hernandez, C., Page, M., Sari, T., Geiselmann, J.: Hybrid modeling and simulation of genetic regulatory networks: A qualitative approach. HSCC'2003, A. Pnueli, O. Maler (eds.), LNCS 2623, Springer-Verlag, Berlin, pp. 267$282,(2003)$

8. Demongeot, J., Aracena, J., Thuderoz, F., Baum, T.-P., Cohen, O.: Genetic regulation networks: circuits, regulons and attractors. C. R. Biologies 326, (2003)

9. Edwards, R., Glass, L.: Combinatorial explosion in model gene networks. Chaos 10 (3), 691-704 (2000) 
10. Edwards, R.: Analysis of continuous-time switching networks. Physica D 146, 165-199 (2000)

11. Edwards, R.: Chaos in neural and gene networks with hard switching. Differential Equations and Dynamical Systems 9, 187-220 (2001)

12. Edwards, R., Siegelmann, H.T., Aziza, K., Glass, L.: Symbolic dynamics and computation in model gene networks. Chaos 11 (1), 160-169 (2001)

13. Edwards, R., MacDonald, J.J., Tsatsomeros, M.J.: On matrices with common invariant cones with applications in neural and gene networks. Linear Algebra and its Applications 398, 37-67 (2005)

14. Farcot, E.: Transitions d'états dans un réseau génétique affine par morceaux. technical report available at http://www-lmc.imag.fr/lmc-cf/Etienne.Farcot/Text.html (in French), 2003

15. Fu, X.-C., Lu, W., Ashwin, P., Duan, J.: Symbolic representation of iterated maps. Topological Methods in Nonlinear Analysis 18, 119-147 (2001)

16. Gedeon, T.: Global dynamics of neural nets with infinite gain. Physica D 146, 200-212 (2000)

17. Gedeon, T.: Attractors in continuous time switching networks. Communications on Pure and Applied Analysis (CPAA) 2 (2), 187-209 (2003)

18. Glass, L., Kauffman, S.: The logical analysis of continuous non-linear biochemical control networks. J. Theor. Biol. 39, 103-129 (1973)

19. Glass, L.: Classification of biological networks by their qualitative dynamics. J. Theor. Biol. 54, 85-107 (1975)

20. Glass, L.: Combinatorial and topological methods in nonlinear chemical kinetics. J. Chem. Phys. 63, 1325-1335 (1975)

21. Glass, L., Pasternack, J.S.: Prediction of limit cycles in mathematical models of biological oscillations. Bull. Math. Biol. 40, 27-44 (1978)

22. Glass, L., Pasternack, J.S.: Stable oscillations in mathematical models of biological control systems. J. Math. Biol. 6, 207-223 (1978)

23. Gouzé, J.L., Sari, T.: A class of piecewise linear differential equations arising in biological models. Dynamical systems 17, 299-316 (2003)

24. Henk, M., Richter-Gebert, J., Ziegler, G.M.: Basic properties of convex polytopes. In: CRC Hanbook of discrete and computational geometry, J.E. Goodman, J.O'Rourke, (eds.), Boca Raton, New York, CRC Press, 1997

25. Hofer, J.E.: Topological entropy for noncompact spaces. Michigan Math. J. 21 (3), 235-242 (1975)

26. Kappler, K., Edwards, R., Glass, L.: Dynamics in high-dimensional model gene networks. Signal Processing 83, 789-798 (2003)

27. Killough, D.B., Edwards, R.: Bifurcations in Glass networks. Int. J. Bifurcation Chaos 15, 395-423 (2005)

28. Kauffman, S.A.: The origins of order. Oxford University Press, 1993

29. Lewis, J., Glass, L.: Steady states, limit cycles, and chaos in models of complex biological networks. Int. Jour. Bif. Chaos 1, 477-483 (1991)

30. Lewis, J.E., Glass, L.: Nonlinear and symbolic dynamics of neural networks. Neural Computation 4, 621-642 (1992)

31. Lind, D., Marcus, B.: An introduction to symbolic dynamics and coding. Cambridge University Press, 1995

32. Mestl, T., Plahte, E., Omholt, S.W.: Periodic solutions of piecewise-linear differential equations. Dyn. Stab. Syst. 10 (2), 179-193 (1995)

33. Mestl, T., Lemay, C., Glass, L.: Chaos in high-dimensional neural and gene networks. Physica D, 98, 33-52 (1996) 
34. Mestl, T., Bagley, R.J., Glass, L.: Common chaos in arbitrarily complex feedback networks. Physical Review Letters 79 (4), 653-656 (1997)

35. Misiurewicz, M.: On Bowen's definition of topological entropy. Discrete Contin. Dyn. Syst., Ser. A 10, 827-833 (2004)

36. Plahte, E., Mestl, T., Omholt, S.W.: A methodological basis for description and analysis of systems with complex switch-like interactions. J. Math. Bio. 36, 321-348 (1998)

37. Plahte, E., Kjøglum, S.: Analysis and generic properties of gene regulatory networks with graded response functions. Physica D 201, 150-176 (2005)

38. Snoussi, E.H.: Qualitative dynamics of piecewise-linear differential equations: a discrete mapping approach. Dyn. Stab. Syst. 4 (3-4), 189-207 (1989)

39. Thom, R.: Modèles mathématiques de la morphogenèse. Bourgois, 10-18, 1974, réed., 1980

40. Thomas, R., D’Ari, R.: Biological Feedback. CRC-Press, Boca Raton, Florida, 1990

41. Ziegler, G.M.: Lectures on polytopes. Graduate Texts in Mathematics 152, SpringerVerlag, New York, 1995 